Vol. 22, $n^{\circ} 1 \mid 2018$

Varia

\title{
Discipliner par la grâce. Les lettres de rémission aux gens de guerre dans l'État bourguignon au $\mathrm{XV}^{\mathrm{e}}$ siècle
}

Quentin Verreycken

\section{(2) OpenEdition Journals}

Édition électronique

URL : http://journals.openedition.org/chs/2113

DOI : $10.4000 /$ chs. 2113

ISSN : 1663-4837

Éditeur

Librairie Droz

Édition imprimée

Date de publication : 31 octobre 2018

Pagination : 5-32

ISSN : 1422-0857

\section{Référence électronique}

Quentin Verreycken, « Discipliner par la grâce. Les lettres de rémission aux gens de guerre dans l'État bourguignon au XVe siècle ", Crime, Histoire \& Sociétés / Crime, History \& Societies [En ligne], Vol. 22,

$\mathrm{n}^{\circ} 1$ | 2018, mis en ligne le 31 décembre 2020, consulté le 12 janvier 2021. URL : http://

journals.openedition.org/chs/2113; DOI : https://doi.org/10.4000/chs.2113 


\title{
Prix Herman Diederiks Discipliner par la grâce \\ Les lettres de rémission aux gens de guerre dans l'État bourguignon au XVe siècle
}

\author{
Quentin Verreycken'
}

\begin{abstract}
Cet article traite de l'usage du droit de grâces par les ducs de Bourgogne au $X V^{e}$ siècle en tant qu'outil disciplinaire. Plus spécifiquement, il s'intéresse aux lettres de rémission accordées aux soldats. En comparant le contenu de ces grâces avec les normes prescrites par la législation militaire, cette recherche envisage la possibilité d'un "gouvernement par la grâce» des armées bourguignonnes. L'incrimination des formes les plus extrêmes de la brutalité militaire, ainsi que les nombreuses grâces délivrées aux combattants qui faisaient un usage modéré et légitime de la violence, suggèrent que les ducs de Bourgogne cherchèrent à promouvoir l'image idéalisée d'un soldat professionnel et obéissant, loyal serviteur de l'État.
\end{abstract}

This article seeks to investigate the use of the power to pardon by the dukes of Burgundy as a disciplinary tool in the fifteenth century. More specifically, it focuses on the remission letters granted to soldiers. By comparing the contents of these pardons with the norms elaborated by the military legislation, this study envisages the possibility of a "government by pardoning" towards the Burgundian armies. The criminalization of the most extreme forms of military brutality, together with the numerous pardons delivered to the combatants who had a moderated and legitimated use of violence, suggest that the dukes tried to promote the idealized image of a professional, obedient soldier, as a loyal servant of the State.

\section{INTRODUCTION}

«Tu t'en vas en la guerre, mais ce n'est que pour desrober!». L'invective, prononcée au début l'été 1457 par un certain Jaquemart Frappart, ne laisse guère de doute sur le fait que son auteur ne portait pas les gens de guerre dans son cœur. Il ajoute même que «c'estoit dommage que [...] tous ceulx qui aloient en la guerre n'estoient pendus ». Le destinataire de ces insultes, un habitant de Valenciennes prénommé Jaquemart de le Croix, faisait partie d'un contingent de

1 Quentin Verreycken est historien, Belgian American Educational Foundation Postdoctoral Fellow à l'Université Harvard et docteur de l'Université catholique de Louvain (Centre d'histoire du droit et de la justice) et de 1'Université Saint-Louis - Bruxelles (Centre de recherches en histoire du droit et des institutions). Sa thèse de doctorat est une étude comparée des grâces accordées aux soldats en Angleterre, France et État bourguignon au XVe siècle (2018). Il a notamment publié Verreycken (2014, 2017a, 2017b). 
miliciens flamands levés par les villes d'Onnaing et de Quarouble pour aller servir dans la garnison de Termonde. L'homme de guerre n'était pas aux bouts de ses peines: quelques jours plus tard, il fut apostrophé par les deux fils de Jaquemart Frappart, qui «lui disoient grandes iniures et villenies, en jurant orribles sermens qu'il seroit batuz». Jaquemart de le Croix eut beau leur répondre qu'il se rendait au service du duc de Bourgogne «pour eulx mesmes et pour tous ceulx desdites villes», il ne put éviter une rixe au cours de laquelle l'un de ses assaillants fut tué. Après s'être absenté du pays afin de se soustraire à la justice, il adressa une demande de grâce au duc de Bourgogne Philippe le Bon, lequel lui octroya sa lettre de rémission en mars $1458^{2}$.

Cet épisode, somme toute classique, de sociabilité conflictuelle s'achevant par un acte de violence, ressemble à des centaines d'autres qu'il est possible de rencontrer dans les lettres de rémission du XIV au XVI ${ }^{e}$ siècle ${ }^{3}$. Cependant, ce cas n'en est pas moins révélateur des relations difficiles que pouvaient entretenir, à la fin du Moyen Âge, les gens de guerre avec ceux que l'on nommera par commodité les «noncombattants ${ }^{4} »$. Les railleries de Jaquemart Frappart rappellent, dans la littérature politique, les dénonciations répétées des excès commis par les gens d'armes depuis le début de la guerre de Cent Ans, à l'instar de ce passage de la Ballade contre les gens de guerre (v. 1396) d'Eustache Deschamps: «li mandez destruisent leur contrée, prannent, pillent quanqu'ilz puelent trouver, sanz riens paier; l'eglise est violée, mainte chose est chascun jour ransonnée, et l'un pais l'autre voisin destruit; [...] telz gens se font gens d'armes appeler ${ }^{5}$.» À l'inverse, la réponse de Jaquemart de le Croix fait appel aux valeurs du service au Prince et à la défense du bien commun, développées sous la plume des théoriciens du pouvoir et de la chose militaire à partir du milieu du XIV siècle $^{6}$. En vis-à-vis de l'image négative du « soldat-tyran ${ }^{7}$ », s'est construite l'idée du métier des armes comme d'une charge publique telle que théorisé par Jean de Salisbury dans son Policraticus $(1159)^{8}$ et reprise trois siècles plus tard par l'auteur du Jouvencel (v. 1461-1466), qui compare l'office de justice avec l'office des armes: «il doit avoir gens pour la justice et hommes pour la guerre, et [ne] doit estre une mesme chose ${ }^{9}$.» Ces deux visions de l'homme de guerre, en apparence antinomiques, sont en fait caractéristiques des transformations que connaissent les structures militaires en Europe occidentale à la fin du Moyen Âge.

La volonté de réformer le métier des armes, telle qu'elle apparait à partir du $\mathrm{XIV}^{\mathrm{e}}$ siècle dans la littérature politique mais également dans la règlementation militaire des principaux belligérants de la guerre de Cent Ans (France, Angleterre et principautés bourguignonnes), est fondée à la fois sur la condamnation légale

Archives départementales du Nord (ADN), B 1689 fol. 6 v.-7 r., également citée par Petit-Dutaillis (1908, p. 25-26) et Toureille (2006, p. 159).

3 Arnade, Prevenier (2015); Gauvard (1991); Muchembled (1989); Paresys (1998).

4 Allmand (1971).

5 Deschamp (vol 1, 1878, p. 160). Pour une analyse des discours sur la violence des gens de guerre aux $\mathrm{XIV}^{\mathrm{e}}$-XVe siècles, voir Fargette (2007); Gauvard (2000); Saul (2002).

6 Allmand (1991); Wright (1976).

7 La tyrannie des gens de guerre envers la population est un motif que l'on retrouve chez plusieurs auteurs du début du $\mathrm{XV}^{\mathrm{e}}$ siècle, cf. Bove (2010).

8 Lachaud (2010, p. 207-214).

9 Le Jouvencel (vol. 2, 1889, p. 14). 
et morale du mauvais comportement des combattants et sur la transformation de leur rôle, ceux-ci étant de plus en plus envisagés comme des soldats professionnels au service de l'État et du bien commun ${ }^{10}$. À cette époque, les armées médiévales ne sont pas encore ces institutions «totales» et «disciplinaires» qui ne prennent véritablement leur essor qu'au XVII ${ }^{e}$ siècle ${ }^{11}$. Pourtant, entre le début du XV et la fin du XVI ${ }^{\mathrm{e}}$ siècle, commence à se mettre en place dans de nombreux États européens un processus «whereby small, decentralized, self-equippedfeudal hosts were replaced by increasingly large, centrally financed and supplied armies that equipped themselves with ever more sophisticated and expensive weaponry ${ }^{12}$. Cette «révolution militaire», comme l'appellent Michael Jones et Geoffrey Parker, semble être ellemême étroitement corrélée à la formation des États modernes bureaucratiques ${ }^{13}$. Dans le paradigme de la révolution militaire, l'impératif wébérien de monopolisation de la violence légitime par l'État, rendu possible par le durcissement de la législation et le renforcement du pouvoir des institutions judiciaires, doit également permettre un meilleur contrôle des gens de guerre ${ }^{14}$. Pour l'historien allemand Gerhard Oestreich, ce renforcement de la coercition étatique à l'égard des combattants entraîne la «disciplinarisation sociale» (Sozialdisziplinierung) de ces derniers, ce qui permet notamment le développement du «drill», cette discipline militaire censée assurer la cohésion des troupes sur le champ de bataille tout en favorisant un usage optimal de leurs armes ${ }^{15}$. Á ceci vient s'ajouter, à partir de la seconde moitié du XVI $\mathrm{I}^{\mathrm{e}}$ siècle, le durcissement de l'encadrement moral et religieux des gens de guerre, rendu nécessaire par la Réforme et la Contre-Réforme ${ }^{16}$.

Au moment où Jaquemart de le Croix requiert sa lettre de rémission, en 14571458, les armées des ducs de Bourgogne sont encore constituées en majorité de troupes levées pour une courte durée, via la convocation du ban et de l'arrière-ban. Mais, quelques années plus tard, on peut assister à une première transformation des institutions militaires de l'État bourguignon au cours du principat de Charles le Hardi (1467-1477), dit le Téméraire, établissant des armées permanentes nommées les «compagnies d'ordonnance ${ }^{17} »$. Cette réforme se caractérise également par l'accroissement, dans la législation militaire, des dispositions répressives à l'égard des crimes commis en l'armée, ainsi que par le renforcement du pouvoir de la justice militaire par l'intermédiaire du maréchal de Bourgogne et du prévôt des maréchaux ${ }^{18}$. Malheureusement, les archives de cette justice militaire sont pour ainsi dire inexistantes avant le début du XVI ${ }^{\mathrm{e}}$ siècle dans les anciens Pays-Bas ${ }^{19}$. Il n'en va pas de même des lettres de rémission accordées aux gens de guerre, qui se révèlent relativement nombreuses, au point de représenter près d'un cinquième de l'activité

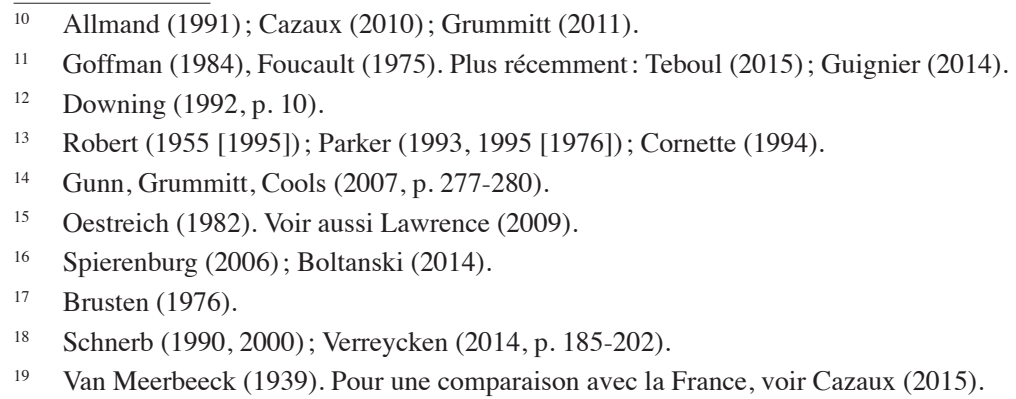


miséricordieuse des ducs de Bourgogne au $\mathrm{XV}^{\mathrm{e}}$ siècle. Se pose alors la question d'une éventuelle forme d'impunité des combattants : en graciant à répétition les gens de guerre, les monarques font-ils preuve de laxisme à leur égard?

Pardonner les combattants est une pratique courante des monarques de la fin du Moyen Âge. On la rencontre particulièrement dans les royaumes de France et d'Angleterre ${ }^{20}$, ainsi qu'au Portugal ${ }^{21}$, de même que dans certaines principautés comme le duché de Lorraine ${ }^{22}$. Dans le présent article, nous étudierons un cas particulier, celui des lettres de rémission accordées à des soldats par les ducs de Bourgogne. En nous appuyant sur un corpus d'un peu plus de 150 lettres de rémission, nous tâcherons ainsi d'observer quelle pouvait être la ou les politique(s) princière(s) de grâce. Pour ce faire, nous mettrons en perspective le contenu de ces lettres avec les prescriptions de la législation militaire, afin d'observer les contradictions ou au contraire les convergences entre l'incrimination primaire de la violence des soldats et le traitement effectif de cette dernière par la justice retenue du prince. Il s'agira ainsi de mette en lumière ce que nous identifions comme un programme disciplinaire des ducs de Bourgogne, destiné à promouvoir une image idéalisée du soldat et visant, de cette façon, à réformer les armées par une série de prescriptions normatives.

\section{PARDONNER L'HOMME DE GUERRE AU XVe SIÈCLE}

La fin du XIV siècle et le début du XVe siècle voient l'émergence d'un «État bourguignon » dirigé par la dynastie des ducs de Bourgogne de la maison Valois, fruit du rassemblement disparate des principautés des anciens Pays-Bas avec le duché et le comté de Bourgogne - ce que Robert Stein désigne comme une «monarchie composite $^{23} »$. Dès l'accession de Philippe le Hardi au titre de comte de Flandre, d'Artois et de Bourgogne en 1383, cet Etat est fondé sur le développement d'une administration ducale forte - les Conseils de justice et les Chambres des comptes - et sur l'affirmation du pouvoir souverain du duc, y compris en tant que juge suprême de ses principautés et donc détenteur du droit de gracier les criminels en leur accordant une lettre de rémission ${ }^{24}$.

Pour paraphraser la définition qu'en donne Claude Gauvard, la lettre de rémission est un acte de chancellerie par lequel un roi ou un prince - ici le duc de Bourgogne - octroie son pardon à la suite d'une infraction, suspendant le processus ordinaire de la justice ${ }^{25}$. Elle est délivrée à la suite d'une requête remise par le demandeur à la chancellerie ducale. Celle-ci est ensuite transmise au duc de Bourgogne et à son conseil par l'entremise d'un maître des requêtes. Le monarque et ses conseillers examinent en détail la supplique qui décrit le crime commis, en tentant de donner les raisons pour lesquelles le suppliant méritait d'être gracié. Si le cas est jugé rémissible, le duc de Bourgogne fait expédier une lettre de rémission,

\footnotetext{
20 Gauvard (2009); Prétou (2010); Lacey (2009); Villalon (2013).

21 Cruz Coelho (2001).

22 Pégeot, Derniame, Hénin (2013).

23 Stein (2017, p. 12-13). Sur le débat historiographique autour de la notion d'État (ou «des états») bourguignon, voir Schnerb (1990); Cauchies (2010): Lecuppre-Desjardin (2016).

24 Arnade, Prevenier (2015); Beaulant (2015); Boone (1989); de Schepper (1995).

25 Gauvard (1991, p. 63).
} 
que le suppliant doit finalement faire entériner par une juridiction locale. Du point de vue de la diplomatique, le texte de chaque lettre de rémission est divisé en deux grandes parties: d'une part, l'exposé des motifs, destiné à justifier la décision du monarque, mais qui n'est en fait qu'une reprise de la requête de grâce décrivant le crime commis, et le dispositif qui contient les formules juridiques du pardon, d'autre part.

Les descriptions contenues dans la première partie des lettres de rémission prennent le plus souvent la forme d'un court récit narrant les circonstances qui ont amené le suppliant à commettre son crime: disputes de taverne, rixes de rue, jeux qui tournent mal, etc. Parce qu'ils recueillent le discours d'hommes et de femmes issus d'à peu près toutes les couches de la population et particulièrement les individus de condition modeste, les récits de rémission semblent être une source presque inépuisable pour l'étude des mœurs et des conflits propres aux sociétés du passé26. Mais le caractère parfois très vivant de ces textes ne doit pas faire oublier qu'ils sont issus d'une demande formellement adressée au souverain en vue d'obtenir la grâce. Les récits de rémission appartiennent ainsi à un genre de documents bien précis, les «suppliques» (aussi appelées petitions, gravamina, supplikationen, etc. ${ }^{27}$ ). Celles-ci se caractérisent par leur fonction (convaincre l'autorité destinataire du bien-fondé de la demande qui lui est adressée) ainsi que par les règles (rhétoriques, diplomatiques, juridiques) de rédaction auxquelles elles sont soumises ${ }^{28}$. Les suppliques de rémission contiennent par exemple ce que Pascal Texier appelle des «stratégies de disculpation» qui visent à atténuer la responsabilité du suppliant, en reportant la faute sur la victime ou sur un mauvais coup du sort ${ }^{29}$. À ceci s'ajoute le fait que le contenu de ces requêtes est généralement le produit de plusieurs intervenants - à commencer par les clercs de chancellerie chargés de recueillir le témoignage du suppliant -, bien informés sur le fonctionnement de la procédure de grâce ainsi que sur les attentes du pouvoir ${ }^{30}$.

Mais, si les suppliants disposent d'une certaine latitude pour embellir leur récit, ils ne peuvent pas, pour autant, se permettre de livrer un témoignage entièrement mensonger du fait de l'existence de certains moyens de contrôle. D'une part, il est fréquent que le monarque et son conseil décident, avant d'octroyer ou non la rémission, de commissionner une juridiction inférieure afin qu'elle enquête sur l'affaire et livre un «avis» ou une «information préparatoire ${ }^{31} »$. D' autre part, durant l'entérinement la partie victime peut contester les déclarations du suppliant, ce qui, au terme d'une nouvelle enquête, risque d'amener à l'annulation de la lettre au motif qu'elle est « subreptice» et «obreptice ${ }^{32} »$. Pour espérer être gracié, il faut donc que le

$26 \quad$ Vaultier (1965); Bourin, Chevalier (1981); Ulbricht (1996); Würgler (2002).

27 Pour un état de la question, voir notamment Van Voss (2002); Nubola et Würgler (2002, 2006) ; Maillard-Luypaert (2003); Millet (2003); Dodd (2007); Bercé (2014). L'historiographie allemande de la Supplikenforschung a particulièrement fait l'objet d'une synthèse récente dans Hausmann, Schreiber (2015, p. 71-74).

28 Würgler (2002, p. 32-34). Sur les règles implicites et explicites de rédaction des suppliques, voir notamment Davis (1988); Fassin (2000); Dodd (2011); Verreycken (2017b).

29 Texier (2001, p. 487).

30 Musin, Nassiet (2010, p. 56). Voir aussi Gauvard (1997); Davis (1988, p. 55).

31 Vrolijk (1999); Gauvard (2008).

32 Vrolijk (2001, p. 381-384, 393-406). 
suppliant s'inscrive dans un «espace de crédibilitée ${ }^{33} »$ qui ne s'éloigne pas trop de la vérité. C'est la raison pour laquelle, s'il faut traiter avec prudence certains éléments des récits de rémission - particulièrement ceux portant sur le caractère intentionnel ou non du crime commis - en les considérant avant tout comme des arguments juridiques et rhétoriques, les situations présentées dans les textes demeurent, de façon générale, plausibles.

À partir de septembre 1386, la majorité des lettres octroyées par les ducs de Bourgogne sont conservées dans les registres de l'audience de la Chambre des comptes de Lille. Ceux-ci forment un ensemble de 145 volumes allant jusqu'en 1661. En mars 1482, à la mort de Marie, dernière duchesse de la dynastie des Valois-Bourgogne, environ 885 copies de lettres de rémission se trouvent partiellement ou entièrement retranscrites dans les registres, malgré une série de trous noirs archivistiques, dont le plus important couvre les années 1393-1438. Ce total inclut un peu moins d'une trentaine de lettres d'abolition, qui se distinguent des rémissions par le fait qu'au travers d'une sorte de fiction juridique, elles effacent jusqu'au souvenir du crime commis. À ce titre, les abolitions concernent généralement des cas en théorie irrémissibles portant atteinte à la majesté du monarque ${ }^{34}$. En bref, si cet ensemble documentaire de près de 900 actes paraît d'une taille importante, il n'en reste pas moins modeste comparé aux dizaines de milliers de lettres de rémission et de pardon délivrées le roi de France ou d'Angleterre au cours des $\mathrm{XIV}^{\mathrm{e}}$ et $\mathrm{XV}^{\mathrm{e}}$ siècles ${ }^{35}$.

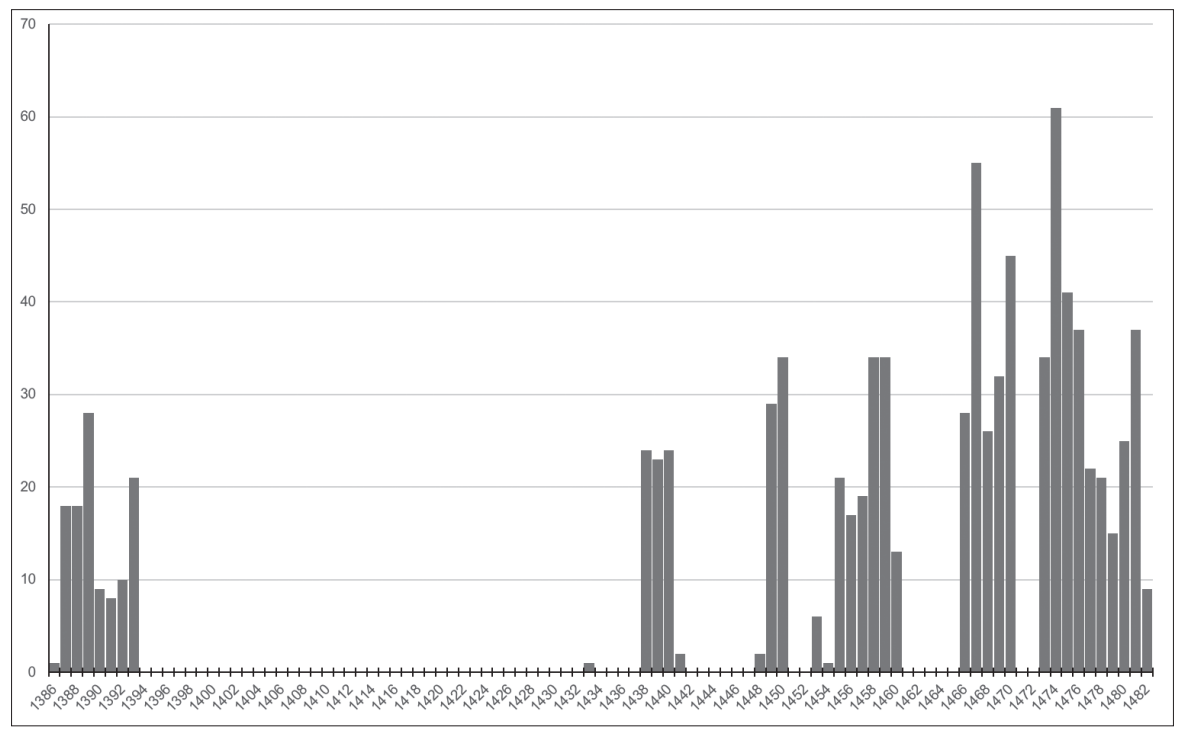

Figure 1. Les lettres de rémission des registres de l'audience (septembre 1386-mars 1482).

\footnotetext{
33 Fassin (2000, p. 964).

34 Pour plus d'informations sur les différents types de grâces: Dauven (2014); Verreycken (2014, p. 53-55).

35 Gauvard (1991); Lacey (2009).
} 
Grâce à différents indices figurant dans les lettres de rémission, on peut estimer que 157 des 885 grâces conservées dans les registres de l'audience, soit 17,7\% des cas, sont destinées à des individus pratiquant ou ayant pratiqué le métier des armes. Pour identifier les combattants, on peut d'abord se référer à l'exposé des motifs des lettres de rémission, qui débute par la « déclinaison d'identité ${ }^{36}$ » du suppliant donnant son nom et son sexe, ainsi que, selon les cas, d'autres informations comme son âge, sa profession, son domicile ou son lieu de naissance. Si la supplique a été soumise par un homme de guerre, la mention de la profession est généralement remplacée par celle du statut militaire du suppliant, par exemple: «Wiart Poitevin, natif de notre conté de Boullenois, notre archier soubz et en la compaignie de notre amé et

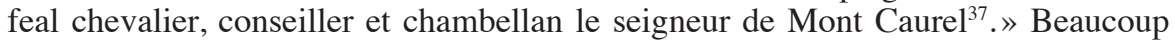
de lettres ne sont toutefois pas aussi précises et utilisent une terminologie plus générique pour évoquer la condition de l'impétrant: «Robert de Bethencourt, dit du Burghel, homme de guerre de la garnison de notre ville d'Aire ${ }^{38}$.» Quelques-unes, au contraire, se montrent particulièrement prolixes et livrent le détail de ses états de service: «icellui suppliant ait depuis le temps de sa jonesse servi feu notre treschier seigneur et père, que Dieu absoille, en toutes ses voyages et armees et depuis son trespas nous, tant en estat d'homme d'armes comme de marchant vendant vins et autres vivres et marchandises soubz et en la compaignie de notre ame et feal cousin le seigneur de Fiennes mareschal de notre host ${ }^{39}$.» Enfin, de nombreuses suppliques se concluent par un court argumentaire résumant les qualités des suppliants et les motifs selon lesquels ils méritent d'être graciés, comme par exemple celui d'avoir servi le duc de Bourgogne dans ses armées: «actendu ce que dit est et que en autres choses icelui suppliant, qui est jeune homme et qui nous a servy en notre derraine armee en France, a tousiours esté de bonne vie, fame, renommee et honneste conversacion, sans oncquesmais avoir esté actaint ne convaincu d'aucun autre villain cas, blasme ou reproche, ainsi qu'il dit, il nous a tres humblement supplié et requis ${ }^{40}$.»

Notons qu'une même lettre de rémission peut être octroyée à plusieurs individus en même temps. À ce titre, les 157 grâces de notre corpus ont bénéficié à 265 gens de guerre clairement identifiés ${ }^{41}$. La majorité des suppliants ne fournissent pas d'indication précise sur leur statut à l'armée: ils se contentent de mentionner qu'ils sont «compaignon de guerre ${ }^{42}$ » ou qu'ils ont «servy en noz guerres et armees ${ }^{43}$ ». On rencontre ensuite des individus se déclarant serviteurs ou valets d'un homme de guerre. Étant donné le caractère assez flou de ces appellations, il n'est pas certain que ces suppliants soient à proprement parler des gens de guerre destinés à combattre

Gauvard (1991, p. 81).

37 ADN, B 1695 fol. 40 r.-41 r. (juillet 1474).

38 ADN, B 1700 fol. 94 r.-v. (décembre 1478).

39 ADN, B 1700 fol. 87 r.-88 r. (mars 1479).

40 ADN, B 1693 fol. 18 v. (octobre 1467).

41 De ce total sont exclus 13 co-bénéficiaires n'étant pas identifiés comme combattants. Il faut également signaler deux lettres destinées à un groupe plus ou moins étendu d'individus, mais sans qu'en soit précisé le nombre exact: d'une part, les «maistres et manouviers de la flote des nefs d'Espaigne» (ADN, B 1681 fol. 10 v.-11 r., janvier 1387), et d'autre part, «aucuns capitaines, gens de guerre et autres » du duc de Gueldre (ADN, B 1693 fol. 89 v.-99 r., juillet 1469). Le nombre exact d'individus composant ces groupes n'étant pas précisé, nous ne les avons pas pris en compte dans notre total.

42 ADN, B 1703 fol. 34 v.-35 r. (novembre 1481).

43 ADN, B 1691 fol. 114 r.-116 v. (novembre 1466). 


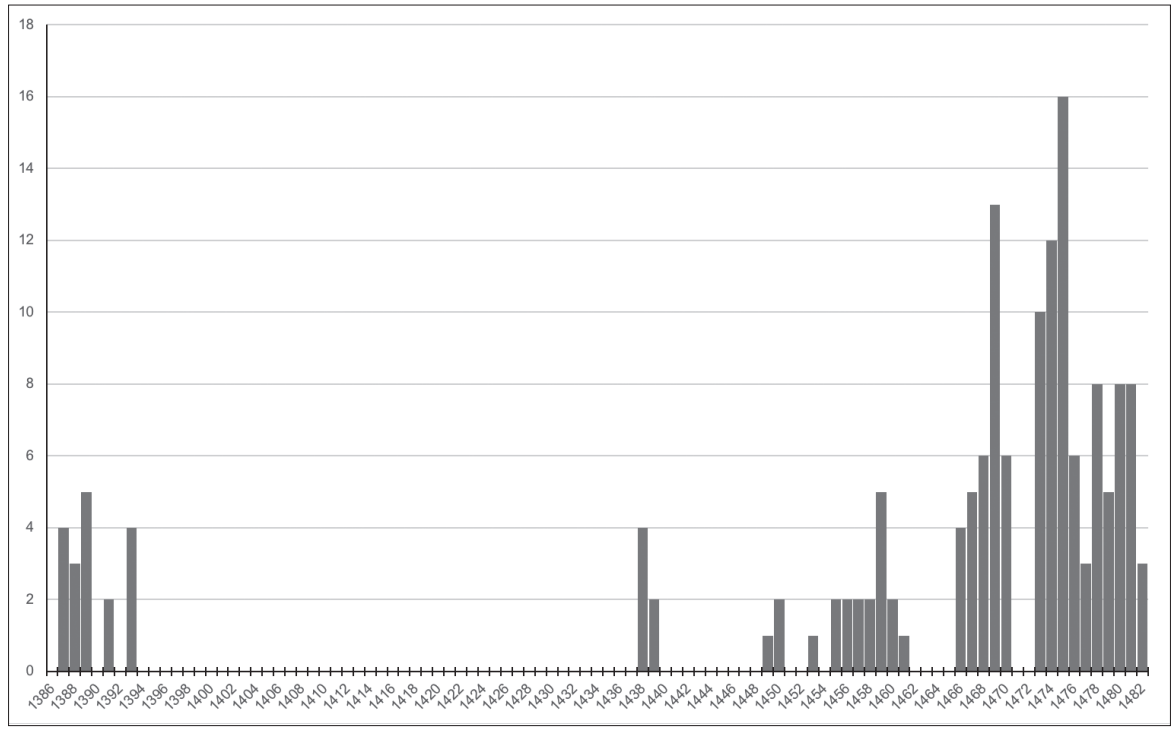

Figure 2. Les lettres de rémission aux combattants (septembre 1386-mars 1482).

sur le champ de bataille, mais cela ne les empêche pas de régulièrement revendiquer avoir «exposé leurs corps ${ }^{44}$ » au service du duc de Bourgogne. Ils peuvent donc être considérés comme faisant partie intégrante des armées. Derrière les serviteurs, les deux profils les plus fréquemment rencontrés dans les lettres de rémission sont les archers et les hommes d'armes. Certains suppliants affichent par ailleurs plusieurs statuts, tel «Katherin, bastard de Vienne, escuier, homme d'armes et lieutenant de dixainier ${ }^{45}$ » ou Hacquinet de Marquet qui servit successivement le duc «en estat d'archier» puis «en estat d'homme d'armes ${ }^{46}{ }^{\star}$. On peut également signaler que 34 suppliants revendiquent un titre de noblesse, comme écuyer ou chevalier. Il convient toutefois de noter que l'on ne rencontre dans notre corpus aucune mention explicite d'un piquenaire, arbalétrier ou couleuvrinier, lesquels font pourtant partie intégrante des unités de base des armées bourguignonnes. Peut-être ces individus se trouventils parmi les suppliants qui se décrivent comme serviteurs ou compagnons de guerre, sans autre précision sur leur statut?

\footnotetext{
44 ADN, B 1685 fol. 22 r.-23 r. (août 1453).

45 ADN, B 1695 fol. 13 r.-13 v. (octobre 1473).

46 ADN, B 1693 fol. 82 r.-v. (mai 1469).
} 
Table 1. Profils des suppliants combattants $(n=265)^{47}$.

\begin{tabular}{|l|r|r|}
\hline Statut & Nombre & Pourcentage \\
\hline Serviteurs & 35 & 13,2 \\
\hline Archer & 19 & 7,2 \\
\hline Compagnon de guerre & 18 & 6,8 \\
\hline Homme d'armes & 12 & 4,5 \\
\hline Cannonier & 4 & 1,5 \\
\hline Lieutenant de dizainier & 2 & 0,8 \\
\hline Marchand de vivres & 2 & 0,8 \\
\hline Capitaine & 1 & 0,4 \\
\hline Musicien de guerre & 1 & 0,4 \\
\hline Non-précisé & 176 & 66,4 \\
\hline
\end{tabular}

La diversité des profils de nos suppliants permet de souligner que, dans les lettres de rémission, la condition d'homme de guerre est loin de constituer une catégorie socioprofessionnelle homogène. Il importe en outre de garder à l'esprit que, même si les bénéficiaires de la miséricorde ducale sont identifiés comme des combattants dans l'exposé des motifs, ils ne se trouvent pas forcément à l'armée au moment de commettre leur crime. En effet, dans notre corpus, seul un tiers des lettres de rémission (64 sur 157) indiquent clairement que le suppliant vivait du métier des armes au moment où il avait perpétré son méfait. C'est le cas par exemple de Pierot le Roussel, qui débute le récit de son crime en précisant qu'il se trouvait alors «en notre service et armee soubz et en la compaignie de messire Jehan d'Orkainck, chevalier, seigneur de Gemeppre ${ }^{48} \gg$.

À l'inverse, 44 lettres sur 157 sont destinées à des individus qui, bien qu'ils revendiquent leur service de guerre au prince, se sont rendus coupables d'un crime après avoir quitté l'armée. Certaines de ces rémissions ne s'inscrivent donc plus à proprement parler dans un contexte militaire et ne peuvent pas être analysées comme des exemples de violences perpétrées par des gens de guerre. D'autres, au contraire, se révèlent indicatrices des situations auxquelles peut être confronté un ancien soldat tentant de se réintégrer dans la société. Gilles Potel, par exemple, pardonné pour avoir commis divers vols, motive sa supplique en exposant qu'il «nous a bien et loyalment servy en noz guerres, voyaiges et armees et que a l'occasion des grands pertes et dommaiges qu'il a euz et soustenuz en notredit service il a esté contraincte par poureté de faire et commectre les cas et larrcins qui s'ensuivent ${ }^{49} \gg$.

Une troisième catégorie de lettres (39 cas) concerne les bénéficiaires ayant commis leur crime avant de rejoindre l'armée. Ainsi, le cordonnier Jehan de Biannel, après avoir occis Petit Jehan Buteux, «s'est tousjours depuis tenu absent de noz pays de Picardie jusques a environ a ung an qu'il a esté receu en nosdites ordonnanes

47 Le total dépasse légèrement les $100 \%$ puisque quelques suppliants renseignent plusieurs statuts.

48 ADN, B 1703 fol. 34 v.-35 r. (novembre 1481).

49 ADN, B 1687 fol. 23 r.-24 r. (mai 1457). 
en estat d'archier ${ }^{50}$ ». Étant donné la portée géographique limitée des juridictions locales, fuir le lieu du méfait est un bon moyen pour un criminel d'échapper à la justice $^{51}$. La fuite risque toutefois d'entraîner une forme de déracinement social pour le coupable, comme l'expriment souvent dans les rémissions les suppliants qui affirment «vivre en estrange marche et contree en grant poureté et misere ${ }^{52}$ ». Rejoindre l'armée permet dès lors aux fugitifs de refaire leur vie et de subvenir à leurs besoins.

La quatrième catégorie de lettres, la plus rare de notre corpus (trois cas seulement), consiste en des lettres délivrées à des non-combattants, à conditions que ceux-ci prestent un service de guerre au duc de Bourgogne, à l'instar de Marcille Hella, «tenu de nous servir en ceste notre presente armé que faisons a l'encontre de ceulx de la ville et chastellenie de Dynant ainsi qu'il dit qu'il en a intencion et voulenté ${ }^{53} \gg$.

Soulignons, pour finir, que toutes les lettres de rémission ne se montrent malheureusement pas aussi précises que celles qui viennent d'être évoquées. Sept actes signalent en effet le service de guerre du suppliant, mais ne livrent aucun indice sur le fait qu'il ait pu commettre son crime avant, pendant ou après sa vie à l'armée. Ils forment ainsi une cinquième et dernière catégorie de lettres du corpus.

\section{"METTRE EN ORDRE ET DISCIPLINE »: LA LÉGISLATION MILITAIRE BOURGUIGNONNE}

En 1473, le duc de Bourgogne Charles le Hardi promulgue à l'abbaye de SaintMaximin, près de Trèves, ce que d'aucuns ont jadis considéré comme le «plus beau code militaire du siècle ${ }^{54} »$. Le règlement, fort de près de soixante articles, prévoie, parfois jusque dans ses moindres détails, l'organisation et la logistique des armées bourguignonnes ${ }^{55}$. Un soin tout particulier est également apporté à l'agencement de chaque item du texte, ce qui contraste avec le caractère souvent brouillon ou désordonnée de la législation militaire de l'époque. L'ambition disciplinaire de l'ordonnance de Saint-Maximin se trouve clairement exprimée dans le préambule du texte: les armées du duc de Bourgogne «ne peuvent être permanentes en obéissance, union et vertueuse opération, sans loy, tant pour leur instruction du debvoir de leurs estats et vocations que pour la promotion de leurs loyaulx et vertueux fais, pugnition et correction de leurs vices et deffaulx », c'est pourquoi le duc a fait établir «les lois, status et ordonnances qui s'ensuivent». Dans la visée de corriger les comportements des gens de guerre, le texte comporte un certain nombre d'articles punissant, souvent de façon sévère, les infractions commises à l'armée. Mais le programme disciplinaire de l'ordonnance de Saint-Maximin ne se limite pas à une simple liste d'interdictions : il est également porteur d'un objectif d'éducation du corps et de l'esprit des soldats. Le cinquantième article du règlement instaure un système d'entraînement collectif des gens de guerre, avec déplacements en formation, exercices de tir, manœuvres

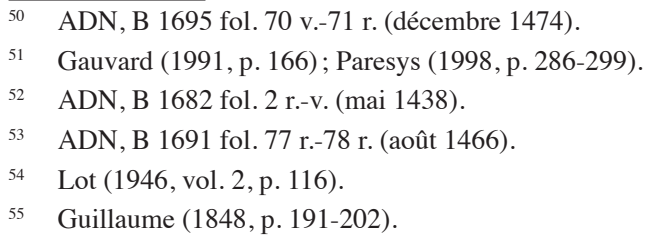


offensives et défensives, bref, une certaine forme embryonnaire de drill ${ }^{56}$. Le conducteur de chaque compagnie, chargé de diriger ces entraînements, doit ainsi enseigner aux soldats «l'amour et obéissance qu'ils doibvent avoir envers mondit seigneur». Ce faisait, son rôle est de «les mettre en ordre et discipline ${ }^{57} »$.

Si l'ordonnance de 1473 marque probablement l'apogée de la science militaire dans l'État bourguignon, elle n'en est pas moins le produit d'un long processus de renforcement de la coercition à l'égard des combattants, entamé dès le début $\mathrm{du} \mathrm{XV}^{\mathrm{e}}$ siècle. Ainsi, l'octroi croissant de lettres de rémission aux gens de guerre entre 1386 et 1482 se fait en parallèle de la promulgation d'une série de règlements militaires dont les prescriptions, répétées d'un acte à l'autre, permettent l'élaboration d'un corpus normatif portant sur le comportement des gens de guerre ${ }^{58}$. Princes législateurs très actifs en termes de production d'ordonnances et d'édits, les ducs de Bourgogne n'en sont pas moins ce que Jean-Marie Cauchies appelle des «souverains en détail », c'est-à-dire qu'ils dirigent indépendamment chacune de leurs principautés, en y promulguant des règlements qui leur sont propres. Aussi, l'essor d'une législation générale, couvrant l'ensemble des territoires bourguignons, ne se fait que tardivement, dans le dernier quart du $\mathrm{XV}^{\mathrm{e}}$ siècle $^{59}$. Dans cette perspective, les premiers édits réglant la conduite des gens de guerre se révèlent limités à la fois dans l'espace et dans le temps.

En 1417 et 1423, le duc Jean sans Peur puis son fils Philippe le Bon font produire deux plans de bataille contenant quelques articles à dimension disciplinaire. Le premier se limite à punir de mort les gens de guerre qui fuient devant l'ennemi. Le second plan prévoit la peine de mort pour les combattants qui refusent de s'engager dans la bataille, s'enfuient ou font des prisonniers avant la fin de l'affrontement. Il prescrit également une peine à l'arbitraire du capitaine pour les gens de guerre qui provoquaient des disputes ou répandaient des rumeurs parmi les troupes. Comme le note Bertrand Schnerb, «ces mesures ne constituent pas un ensemble homogène de prescriptions répressives; elles ne sont qu'un complément aux mesures tactiques édictées dans des plans de bataille ${ }^{60} \gg$. Nous sommes donc encore loin d'un véritable programme de réforme disciplinaire.

Il faut attendre 1438 pour rencontrer l'ébauche d'une véritable législation répressive vis-à-vis des combattants. À cette époque, le duché de Bourgogne fait face au phénomène de l'écorcherie, c'est-à-dire aux ravages causés par des bandes de gens de guerre démobilisés qui s'en prennent à la population pour survivre ${ }^{61}$. Afin d' «obvier aux grands et énormes dommaiges, pilleries et autres maulx» commis par ces pillards, Philippe le Bon publie le 27 octobre 1438 à Dijon une ordonnance pour le duché, qui promet la pendaison aux combattants qui se rendent coupables de viol, attaque d'église, rançon, incendie ou destruction de maison, en donnant toute la latitude aux baillis pour poursuivre les coupables. Le texte rend en outre ces crimes irrémissibles ( «sans en attendre avoir aucune de par noz grace, misericrode

\footnotetext{
56 Vaughan (1973, p. 209); Verreycken (2016).

57 Guillaume (1848, p. 200-201).

58 Phénomène également observé en France par Cazaux (2010).

59 Cauchies (1981).

60 Scherb (1990, p. 102).

61 Fréminville (1888, p. 89-125).
} 
ne remission $\left.{ }^{62}\right)$. Toujours confronté au phénomène des bandes armées quelques années plus tard, le duc promulgue, en 1445, une nouvelle ordonnance dans le duché de Brabant, autorisant la population à capturer les brigands et à les livrer aux autorités des villes ou des châteaux du duc ${ }^{63}$.

Sous le principat de Charles le Hardi, le nombre de règlements s'accroit rapidement en quelques années, tandis que se mettent en place les principales réformes des structures militaires de l'État bourguignon ${ }^{64}$. En 1468, le duc adresse un ordre au maréchal de Bourgogne afin de lever une armée dans le duché et le comté pour mener la guerre contre la principauté de Liège. L'instruction contient quelques articles disciplinaires prescrivant la peine de mort aux gens de guerre commettant des actes de pillage, rançonnement, violence et mutilation ou viol sur la population des pays de Bourgogne ${ }^{65}$. Au début des années 1470, le Téméraire souhaite revoir en profondeur le fonctionnement de ses armées, afin de disposer d'un instrument militaire à la pointe de la science de la guerre de son époque. Le duc commence par réorganiser le service des fiefs par l'intermédiaire de deux ordonnances promulguées en décembre 1470 et janvier $1475^{66}$. Ensuite, de 1471 à 1476, il publie cinq règlements établissant des armées permanentes, les «compagnies d'ordonnance» (inspirées des régiments du même nom créés par Charles VII en 1445), constituées de combattants de métier recrutés sur base du volontariat. Les textes détaillent l'organisation, la structure et la composition de ces compagnies, et fournissent également un certain nombre de dispositions destinées à maintenir la discipline parmi les troupes, en ciblant les infractions ayant auparavant déjà été condamnées par la législation ducale.

L'une des deux ordonnances d'Abbeville, datant de juillet 1471, sanctionne le départ sans autorisation de la compagnie, d'une perte de quatre jours de gage si l'infraction est commise en pays ami ou d'une punition à l'arbitraire du capitaine en pays ennemi ${ }^{67}$. L'ordonnance de Bohain de novembre 1472 développe ces mesures : quitter la compagnie pour piller ou fourrager est également puni, en pays ami, par la perte de quatre jours de gage, en pays ennemi, par la suspension de ces derniers et la confiscation du cheval et de l'équipement ainsi que, en présence de l'ennemi, par la peine de mort $^{68}$. S'absenter de l'armée sans avoir au préalable obtenu un congé entraîne une peine «criminelle, corporelle ou autre » à l'arbitraire du conducteur de la compagnie. En outre, chaque nouvelle recrue doit désormais prêter un serment de fidélité au duc de Bourgogne, au même titre que n'importe quel autre officier princier $^{69}$. La fameuse ordonnance de Saint-Maximin de novembre 1473 reprend la même liste de punitions que celle de 1472, en y ajoutant l'interdiction du blasphème et des jeux de hasard, sous peine d'une sanction à l'arbitraire du conducteur. L'ordonnance de Lausanne de mai 1476 est le règlement le plus répressif de

62 Champeaux (1912, p. 134-135).

63 Godding (2006, p. 63).

64 On trouvera une synthèse plus détaillée (y compris du point de vue bibliographique) à ce sujet dans Schnerb (1999, p. 269-274); Verreycken (2017a).

65 Chauvelay (1878-1879, p. 144-145); Mémoires pour servir à l’histoire de France et de Bourgogne (vol. 2, 1729, p. 283-285).

66 Schnerb (1999, p. 271-272).

67 Mémoires pour servir à l'histoire de France et de Bourgogne, vol. 2, 1729, p. 293-294.

68 Gollut (1846, col. 1250-1251).

69 Sur le rôle du serment chez les officiers princiers, voir Santamaria (2014). 
Charles le Hardi, car promulguée à un moment où le duc de Bourgogne se trouvait en difficulté, embourbé dans une campagne militaire en Suisse où il enchaîne les défaites. Le texte généralise la peine de mort à pratiquement toutes les infractions: quitter le camp sans autorisation, quitter l'armée lorsqu'elle est rangée en ordre de bataille, piller ou voler en pays ami, commettre un viol ou profaner un lieu saint. Les blasphémateurs sont sanctionnés par une exposition publique pendant un jour et une nuit voire, en cas de récidive, par une peine à l'arbitraire du duc. Enfin, l'ordonnance interdit définitivement la présence de filles de joies à l'armée et, toujours pour limiter les risques d'irruption de conflits parmi les troupes, elle enjoint les conducteurs à ce qu'ils «facent à leurs gens boire plus d'eaue afin qu'ilz soient moins chaulx durant ledit voyage sur ce mestier ${ }^{70} \gg$.

La débâcle des armées bourguignonnes à la bataille de Nancy, le 5 janvier 1477, face aux troupes de René II de Lorraine, et la mort du duc de Bourgogne au cours de l'affrontement, plongent l'État bourguignon dans une crise politique et militaire majeure $^{71}$. Malgré leur échec, les compagnies d'ordonnances sont rapidement rétablies par la duchesse Marie et son mari Maximilien de Habsbourg, même si leur nombre est désormais plus réduit, et les réformes militaires de Charles continuent d'être d'application au moins jusqu'à ce que Charles Quint procède lui-même à la réorganisation des compagnies ${ }^{72}$. Sur le plan règlementaire, on ne rencontre durant le principat de Marie de Bourgogne qu'un seul texte important, malheureusement dépourvu de date mais probablement promulgué peu de temps après la mort du Téméraire: l'ordonnance dite du prévôt des maréchaux. Ce texte règle la conduite des armées en campagne sous l'autorité du maréchal de Bourgogne, en précisant les différentes attributions de l'office du prévôt des maréchaux, du prévôt de l'artillerie ainsi que du contrôleur et du receveur de l'artillerie. Une série d'articles est consacrée aux rôles du maréchal et de son prévôt durant les Joyeuses Entrées, parmi lesquels celui de recueillir les noms des individus susceptibles d'obtenir la grâce du duc de Bourgogne. L'ordonnance précise que «ne doibvent, pour avoir grasce, a savoir boute feus, forceurs de fame, traittres, mordriers et tous ceulx qui sont chargiés de criesme de leesmajesté se n'est qu'il plaise a mondit seigneur ${ }^{73}{ }^{\prime}$. Avec l'ordonnance de 1438, il s'agit donc de l'une des seules traces d'une limitation partielle de la grâce dans l'État bourguignon à la fin du Moyen Âge. Partielle, puisque l'ordonnance précise que le duc pouvait continuer, selon son bon plaisir, à octroyer des lettres de rémission pour ces différents types de crimes. De fait, ce ne sera véritablement qu'au siècle suivant, sous les Habsbourg, que les conditions d'accès à la grâce se sont restreintes de façon drastique ${ }^{74}$.

\section{LA CRIMINALITÉ PARDONNÉE DES GENS DE GUERRE}

Plutôt que de chercher à couvrir tout l'éventail d'infractions susceptibles d'être commises par les gens de guerre - comme le feront plus tard les ordonnances de

\footnotetext{
Chauvelay (1880, p. 325).

71 Sablon du Corail (2016).

72 Guillaume (1873).

73 Schnerb (2000, p. 213).

74 de Schepper (1995); Vrolijk (2004).
} 
l'époque moderne ${ }^{75}$-, la législation militaire des ducs de Bourgogne cible une liste relativement restreinte d'actes qui n'ont eu de cesse de préoccuper le législateur ainsi que l'opinion au cours des derniers siècles du Moyen Âge: désertion, pillage, attaque de la population, etc. À l'inverse, les infractions qui relèvent davantage d'une criminalité plus «ordinaire», au sens où elles ne sont pas spécifiquement associées au métier des armes, sont plutôt du ressort du droit coutumier. En l'absence de conservation des archives des juridictions militaires, il est difficile d'évaluer le degré d'application de ces mesures visant à limiter l'indiscipline des combattants. Plusieurs éléments amènent toutefois à douter de leur succès. Ainsi, les règlements de 1472 et 1473 reprochent aux conducteurs et chefs d'escadre des compagnies d'ordonnance d'être «mols et lasches à faire les pugnitions dessusdictes ${ }^{76} »$. À côté de son activité législative, le duc Charles le Hardi n'aura de cesse de multiplier les mandements adressés à ses officiers de justice afin d'appréhender les déserteurs, visiblement sans beaucoup de succès ${ }^{77}$. Les sources narratives, chroniques ou correspondances épistolaires, attestent en outre des exactions répétées des gens de guerre sur la population, des troubles et des émeutes qui naissent dans les armées ainsi que du taux élevé de désertion en cas de problème d'approvisionnement ${ }^{78}$.

Force est de constater que l'on ne retrouve pas, dans les lettres de rémission accordées aux combattants, les mêmes types d'infractions que ceux couverts par la législation militaire. Du point de vue strictement juridique, l'essentiel de la criminalité pardonnée des gens de guerre ne diffère pas de façon substantielle de celle du reste de la population. On estime que près de $65 \%$ des 885 lettres de rémission conservées dans les registres de l'audience entre 1386 et 1482 concernent des cas d'homicide ${ }^{79}$. Si l'on restreint la recherche aux lettres délivrées aux gens de guerre en service pendant le crime, on constate que ce taux s'élève à près de $80 \%$ des cas (51 lettres sur 64). En outre, la légitime défense, la colère et la vengeance d'un tort passé constituent les motifs les plus souvent invoqués par les suppliants pour justifier leurs actes.

Table 2. La criminalité pardonnée des gens de guerre.

\begin{tabular}{|l|r|r|}
\hline Type de crime & Nombre & Pourcentage \\
\hline Homicide & 51 & 79,7 \\
\hline Coups et blessures & 3 & 4,7 \\
\hline Rébellion & 2 & 3,1 \\
\hline Vol & 2 & 3,1 \\
\hline Autre & 6 & 9,4 \\
\hline Total & 64 & 100 \\
\hline
\end{tabular}

\footnotetext{
75 Guinier (2014, p. 253).

76 Gollut (1846, col. 1253).

77 Cauchies (1977).

78 Viltart (2009).

79 Verreycken (2017b, p. 68).
} 
Alors que, jusque dans les dernières années de la guerre de Cent Ans, Charles VII accorde de nombreuses lettres d' abolition effaçant les diverses «pilleries et roberies ${ }^{80}$ » commises par les combattants, les ducs de Bourgogne n'octroient qu'une seule lettre de ce type, en 1469. Elle est destinée à un groupe de soldats du duc de Gueldre qui avaient attaqué le village d'IJsselstein dans le comté de Hollande, «brulerent ledit villaige, $\mathrm{y}$ tuerent, occirent et navrerent gens et y prindrent et emmenerent plusieurs prisonniers et se pillirent ladicte ville». Pour leur défense, les soldats arguent dans leur supplique qu'ils ignoraient qu'IJsselstein était assujetti à l'autorité du duc de Bourgogne, croyant au contraire qu'il se trouvait dans la principauté d'Utrecht, alors dirigée par le comte d'Egmont, contre lequel le duc de Gueldre était en guerre. De ce fait, ils prétendaient avoir agi «selon la coustume et usaige de guerre ouverte ${ }^{81} »$. À côté de cette abolition, à peine trois autres lettres de rémission accordées par les ducs de Bourgogne concernent des crimes qui peuvent s'apparenter à ce que l'historien Pierre Prétou appelle des «excès de guerre ${ }^{82} »$ : l'attaque d'une abbaye pour s'y loger de force ${ }^{83}$, l'attaque d'une flotte alliée par erreur ${ }^{84}$ et l'incendie d'une ville par les soldats de la garnison craignant qu'elle ne soit prise par l'ennemi ${ }^{85}$.

Il est en réalité difficile de dégager une typologie claire de la criminalité militaire dans les lettres de rémission. En effet, ces dernières privilégient la description exhaustive des circonstances du crime à l'usage d'une terminologie juridique précise. Prenons par exemple la lettre de rémission accordée en février 1388 aux chevaliers Mahieu et Griffon de l'Isques. Le texte décrit comment les deux hommes, «a un certain jour environ l'eure de demie heure devant souleil couchant», tentent de se loger dans une abbaye située près d'Arras, profitant du droit dont jouissent en la matière les gens de guerre au service du roi et du duc de Bourgogne ( «touz gentilz hommes passans le pays en servant le roy en ses guerres et nous aussi es notres povoyent prandre en toutes abbayes et autres lieux logeiz et passage pour une nuit»). Mais face au refus répété du portier de les laisser entrer, les combattants mettent le feu à la porte de l'abbaye, pénètrent dans le couvent, et se battent avec une quinzaine d'individus venus prêter main forte aux moines ${ }^{86}$. Nous sommes donc en présence d'une série d'infractions impliquant un incendie volontaire, l'attaque d'une abbaye, ainsi que des coups et blessures, l'ensemble pouvant en être assimilé à des excès de guerre. Pourtant, dans le dispositif juridique de la lettre, la formule du pardon ducal se contente de se référer «ou cas dessusdit», sans autre précision sur la nature exacte du ou des crimes graciés. En fait, il n'y a que dans les lettres de rémission pardonnant la mort d'homme que l'on peut constater l'usage d'une terminologie juridique un tant soit peu précise, qualifiant l'infraction de «cas et homicide», voire de «cas et murdre» si le geste est commis avec préméditation et dissimulation.

Même si l'essentiel des lettres de rémission accordées à des gens de guerre par les ducs de Bourgogne concerne des affaires d'homicide et non des excès de guerre, ceci ne veut pas dire pour autant que ces grâces sont dépourvues d'intérêt pour

\footnotetext{
$80 \quad$ Gauvard (2009).

81 ADN, B 1693 fol. 89 v.-99 r. (juillet 1469).

82 Prétou (2010, p. 201-202).

83 ADN, B 1681 fol. 47 r.-49 r. (février 1388).

84 ADN, B 1681 fol. 10 v.-11 r. (janvier 1387).

85 ADN, B 1699 fol. 50 r.-51 r. (juillet 1478).

86 ADN, B 1681 fol. 47 r.-49 r. (février 1388). Voir également Schnerb (1996).
} 
l'étude de la sociabilité militaire. Bien au contraire, le contexte dans lequel le crime est commis se révèle souvent indicateur des tensions qui parcourent les armées. Sur la cinquantaine d'homicides commis par des soldats durant leur service, plus de la moitié a également pour victime un combattant, ce qui démontre que la violence des gens de guerre n'est pas uniquement dirigée vers la population.

Parmi les motifs récurrents de conflit, se trouvent d'abord la promiscuité et les problèmes de logement des gens de guerre. Dans les armées bourguignonnes, l'attribution d'un logis pour chaque combattant est la responsabilité du maréchal des logis, assisté par des fourriers qui assurent également l'approvisionnement en vivres $^{87}$. Lorsque les troupes s'arrêtent dans une ville ou un village, c'est lui qui désigne quelles habitations doivent accueillir les gens de guerre. Pour éviter que ces derniers ne rackettent leurs hôtes, les ordonnances militaires régulent l'achat de fournitures auprès des habitants, en prévoyant qu'en cas de vol, l'infracteur devra rembourser sa victime à hauteur du prix de la marchandise volée. Toutefois, des problèmes logistiques peuvent survenir, comme quand trop d'hommes se voient attribuer par erreur le même logis, ou lorsque certains soldats ignorent les ordres et tentent de prendre la maison qui leur plait par la force. En septembre 1466, au cours de la guerre menée contre la ville de Liège par le duc de Bourgogne, l'écuyer Girardin de Herbaumez et plusieurs compagnons sont logés dans la maison de Guillaume Lijsbeth, dans un petit village non loin de Jodoigne. Survient un archer nommé Jehan Passequin qui réclame à son tour d'y avoir sa chambre, ce à quoi le suppliant répond qu'il n'y voit pas d'inconvénient, à condition toutefois que l'archer lui montre son billet de logement donné par le fourrier. À ces mots, Passequin se met à injurier Girardin et à tenter de le frapper d'un gros bâton, ce qui déclenche une rixe au cours de laquelle l'archer trouve la mort ${ }^{88}$. Il arrive également que la population s'oppose à la présence des combattants, comme le narre la lettre de rémission d'Anthonin Journet ${ }^{89}$. En août 1478, l'homme d'armes est logé avec les autres gens de sa compagnie dans un village du comté de Flandre nommé Neuve-Église. Alors que le suppliant est attablé avec son hôte, il entend non loin de là plusieurs villageois en train de déloger certains de ses compagnons, «ne scet icellui suppliant bonnement pourquoy». L'homme d'armes se rend sur place, accompagné de plusieurs autres soldats, et, ensemble, ils assaillent deux villageois et les tuent.

L'historien Robert Muchembled décrivait jadis les pratiques populaires de divertissement dans l'Ancien Régime comme étant «l'antichambre de la violence», soit parce que les jeux permettent de résoudre les conflits latents de manière ritualisée, soit parce qu'au contraire ils peuvent générer de nouvelles tensions susceptibles d'aboutir à des rixes sanglantes ${ }^{90}$. De fait, la vie à l'armée étant marquée par de longues périodes d'attente et d'inactivité, les moments de relâchement apparaissent fréquemment dans les lettres de rémission comme contexte de commission d'un crime $^{91}$. Par exemple, la lettre accordée à l'homme d'armes Emond de Thubeauvile raconte qu'en mai 1473, alors qu'il se trouve en garnison dans la ville de Gueldres, il s'amuse avec plusieurs compagnons à échanger leurs tuniques. Mais lorsque

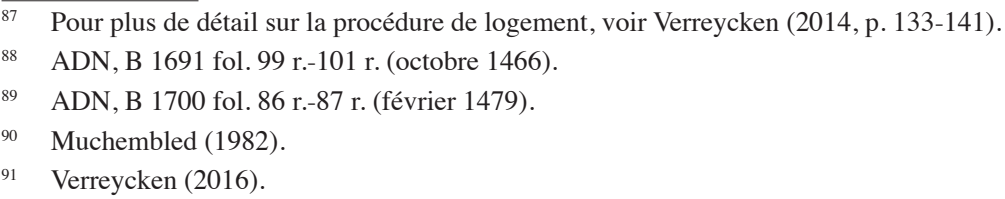


l'un d'eux dit pour plaisanter qu'il voulait faire élargir la manche du vêtement de Guillement de Senecourt, cela provoque la colère de ce dernier. Emond a beau dire à Guillement «qu'il estoit bien folastre de soy courouchier quant on se jouoit alors », celui-ci sort une hachette et s'approche dangereusement du suppliant, lequel réplique d'un coup de dague mortel ${ }^{92}$. La taverne ou l'auberge se trouve également être, on le constate dans une dizaine de lettres de rémission, le théâtre de conflits opposant des gens de guerre à leurs congénères ainsi qu'à des non-combattants. L'alcool, les jeux d'argents et les vieilles animosités aidant, les rixes à la taverne constituent des scènes classiques de violence dans les archives judiciaires d'Ancien Régime ${ }^{93}$. Le conflit survient généralement au moment de régler l'addition ou «escot», lorsque l'un des convives refuse de payer sa part ou met en doute l'honneur et la probité d'une autre personne attablée. Débute alors une dispute s'achevant invariablement par un affrontement au cours duquel l'un ou l'autre parti est tué. Le suppliant se défend toujours d'être à l'initiative de la rixe, rejetant la faute sur la victime : «après pluiseurs parolles furent lesdits trois gros demandez par ledit Pierret audit suppliant pour son escot, a quoy il respondit qu'il les avoit mis sur la table [...] dont eust despit ledit feu Pierret et dit audit supliant qu'il esoit ung broulleur de cela dire, et ledit supliant luy respondit qu'il avoit menty car il n'estoit point broulleur, lors iceluy feu Pierret tira sa daghe pour frapper iceluy suppliant ${ }^{94}$.» Là où les conflits liés au logement des gens d'armes laissent entrevoir des tensions et des rapports de force entre soldats et population liés au contexte militaire, les récits des bagarres de tavernes n'évoquent jamais la condition de combattant ou de non-combattant des belligérants comme un facteur d'opposition, seul l'honneur individuel de chacun étant en jeu.

Un troisième motif courant de conflit est le comportement néfaste d'un combattant faisant mal son devoir, voire s'en prenant à la population, ce que ne manque pas de lui reprocher le suppliant. Ainsi, en décembre 1468, l'homme de guerre Philipe Deschamps voit un combattant nommé Estienne Bouquelet se vanter du fait qu'il rançonne l'hôte chargé de le loger et pille sa nourriture. Le suppliant réagit en maudissant Bouquelet ( «il faiz voz fievres certaines»), lequel lui jette un pot à la figure. Ce geste marque le début d'une rixe au cours de laquelle le pillard est poignardé à mort. Dans le même ordre d'idée, durant le siège de Nimègue en 1473, le canonnier Bauduyn Daublain s'aperçoit que Bernard Wastellet «ne faisoit point son devoir en la charge qui luy avoit esté ordonnee» et ne s'est pas présenté depuis deux jours pour servir au canon. Bauduyn, après lui avoir fait remontrance, le punit en suspendant sa solde. Quelques jours plus tard, les deux hommes se rencontrent à nouveau et une rixe ne tarde pas à éclater, au cours de laquelle Bernard Wastellet trouve la mort ${ }^{95}$. Autre exemple, vers janvier 1478, une quinzaine de soldats viennent "pillier le plat pays et les villes champestres» de Hainaut. Entre autres délits, ils volent les «jumens, vaches, linges et pluseurs autres biens et utensilles» d'une nommée Jehenne Crepin. Apprenant la nouvelle, son frère, l'homme d'armes Phelippe Crepin, vient faire remontrance à certains des pillards, disant «qu'il avoit mal fait d'avoir ainsi pillié et robé les poures paysans

\footnotetext{
$92 \quad$ ADN, B 1695 fol. 2 v.-3 r. (juin 1473).

93 Troch (2012).

94 ADN, B 1695 fol. 72 r.-v. (janvier 1475).

95 ADN, B 1695 fol. 17 v.-18 r. (novembre 1473).
} 
et le plat pays et mesmes d'avoir batu sadite seur». Toutefois, ceux-ci l'attaquent et le blessent à mort. Le suppliant, Hacquinet Crepin, cousin de Phelippe et lui-même ancien homme de guerre, intervient à son tour et donne un coup de dague à François Bronart qui meurt de ses blessures une dizaine de jours plus $\operatorname{tard}^{96}$.

\section{LA RÉSISTANCE DE LA POPULATION}

Si, dans la littérature politique comme dans la législation militaire, les gens de guerre paraissent toujours être les oppresseurs de la population, dans les faits, cette dernière n'est pas entièrement sans défense face aux excès des combattants. Dans le royaume de France, l'ordonnance de 1357 fonde le droit de résistance des sujets face aux attaques des gens de guerre ${ }^{97}$. La Pragmatique Sanction de 1439 promet également d'octroyer rémission pour tout cas de «murdre ou occhision» de combattants qui s'étaient rendus coupables de roberies ou pillages ${ }^{98}$. En réaction au phénomène des groupes de soldats ravageant le pays, on peut ainsi assister à la constitution de bandes de villageois et de paysans armés pourchassant les gens de guerre $^{99}$.

Les principautés bourguignonnes, au $\mathrm{XV}^{\mathrm{e}}$ siècle, sont également confrontées aux compagnies de gens d'armes qui, en période de disette ou lorsqu'elles sont démobilisées, multiplient les pillages et les agressions sur la population. C'est pourquoi Philippe le Bon a, en 1455, promulgué une ordonnance autorisant la population à capturer les pillards pour les remettre aux autorités. De 1386 à 1482, les ducs de Bourgogne ont octroyé 10 lettres de rémission à des non-combattants ayant occis des gens de guerre ${ }^{100}$. Si deux d'entre elles concernent des rixes s'inscrivant dans des conflits de sociabilité somme toute traditionnels (une bagarre suite à une partie de jeu de paumes, ainsi qu'un conflit amoureux autour d'une villageoise) ${ }^{101}$, les huit autres, au contraire, mettent en scène des actes de résistance face aux exactions perpétrées par des soldats sur la population.

Bien loin du modèle positif du «bon soldat», les victimes sont présentées dans ces lettres tour-à-tour comme un «homme de guerre assez mal renommé ${ }^{102}$ », un étranger («les Picars tiennent ma mere, s'elle n'a secours il la destruirons $\left.{ }^{103} »\right)$ ou un inconnu immédiatement soupçonné d'être un ennemi («cuidans de prime face que lesdits compaignons qui estoient en nombre de huit se feussent François $\left.{ }^{104} »\right)$. Ce «mauvais soldat» commet toute la gamme des violences condamnées par la législation militaire, tel que le pillage («ilz firent pluseurs maulx et dommaiges tant

\footnotetext{
$96 \quad$ ADN, B 1699 fol. 41 v.-42 r. (février 1478).

97 Ordonnances des roys de France de la troisième race, vol. 3, 1732, p. 144-145.

98 Crépin (2006, p. 96).

99 Challet (2007).

100 On peut également noter une lettre délivrée à un homme ayant volé les biens d'un soldat, cf. ADN, B 1687 fol. 65 r.-66 v. (janvier 1459).

101 ADN, B 1695 fol. 57 r.-v. (octobre 1474) et fol. 84 r.-85 r. (mai 1475).

102 ADN, B 1700 fol. 82 v.-83 r. (avril 1479).

103 ADN, B 1695 fol. 38 r.-v. (juillet 1474).

104 ADN, B 1699 fol. 45 v.-46 r. (août 1478).
} 
de pillier et fourragier ${ }^{105} »$ ), le viol, y compris sur une femme enceinte ( «veyrent deux compaignons de notre ordonnance de gens d'armes, l'un nommé Jehan Rosseau et l'autre Pernet Boucant; [...] Jehan Rousseau tenoit une espee toute nue sur le corps de ladite femme affin qu'elle voulsist souffrir que ledit Boucant en feist sa volenté, combien que ladite femme lors estoit grosse d'enffant et preste a gesir $\left.{ }^{106} »\right)$, voire des menus larcins ( «il y avoit deux compaignons comme il disoit qui de fait et de force vouloient prendre, ravir et emmener aucuns d'iceulx moutons $\left.{ }^{107} \gg\right)$. Ainsi, en accordant sa grâce aux paysans et aux villageois qui tuent ces pillards, le duc de Bourgogne légitime le droit de résistance de ses sujets face aux excès des soldats ${ }^{108}$.

\section{GOUVERNER LES ARMÉES PAR LA GRÂCE?}

Lorsqu'il décide d'octroyer une lettre de rémission à un requérant, le duc de Bourgogne n'accomplit pas seulement un acte de justice, il fait également acte de gouvernement: il offre au justiciable un moyen d'échapper à la répression de la justice, tout en exigeant la soumission du requérant, qui doit donner de lui l'image d'un sujet humble, fidèle et obéissant ${ }^{109}$. Ainsi, lorsqu'en 1391, Philippe le Hardi accorde sa rémission à l'écuyer Jehan de Follerans pour avoir maintenu le château de Gy, en Franche-Comté, en rébellion contre l'autorité du duc, il impose que le suppliant « jurera judicialement en la main du bailli de notre dict conté de Bourgogne que jamais il ne sera contre nous ou noz successeurs contes de Bourgogne ${ }^{110} \gg$. En outre, en choisissant de gracier davantage certains types de crimes plutôt que d'autres, le monarque dicte également une certaine norme de comportement en désignant les formes de violences licites ${ }^{111}$. Le "gouvernement par la grâce» relève dans cette perspective d'une forme de disciplinarisation des individus ${ }^{112}$. Mais si l'on admet que l'usage du droit de grâce constitue une décision politique, peut-on pour autant conclure que les ducs de Bourgogne firent un emploi particulier de la rémission à l'égard de leurs soldats? Dit autrement, les lettres de rémission octroyées aux gens de guerre au $\mathrm{XV}^{\mathrm{e}}$ siècle peuvent-elles témoigner d'une volonté princière de gouverner et discipliner les combattants en faisant usage de la grâce et, dans l'affirmative, en quoi ce projet consiste-t-il?

Au sommet de leur puissance durant la première moitié des années 1470 , les armées bourguignonnes doivent compter environ 30000 hommes de guerre, ce qui ne représente pas plus d'un pourcent des quelque trois millions d'habitants

\footnotetext{
105 ADN, B 1692 fol. 29 v.-30 r. (janvier 1467).

106 ADN, B 1695 fol. 38 r.-v. (juillet 1474).

107 ADN, B 1699, fol. 46 v.-47 v. (juillet 1478).

108 Prétou (2016).

109 Texier (1984); Beaulant (2016).

110 ADN, B 1681 fol. 104 r.-v. (octobre 1391). Deux autres lettres de rémission sont accordées à des soldats rebelles concernant la même affaire, mais elles n'imposent pas de leur part la prestation d'un serment: ADN, B 1681 fol. 106 r.-v. (janvier 1392) et 114 v.-115 r. (janvier 1393). Pour le contexte de cette affaire: Vaughan (2002, p. 105); Bubenicek (2013, p. 72, 364-434).

111 Gauvard (2005).

112 Gauvard $(1999 ; 2003)$.
} 
des anciens Pays-Bas et des deux Bourgognes ${ }^{113}$. Le fait que 17,7\% des lettres de rémission octroyées entre 1386 et 1482 aient été destinées à des gens de guerre parait donc indiquer que les combattants font l'objet d'une attention particulière de la part des ducs de Bourgogne. Le nombre de lettres délivrées semble lui-même lié au contexte de guerre et aux campagnes militaires menées par les ducs, avec des pics remarquables d'octroi en 1474 et 1475, au moment où Charles le Hardi assiège la ville de Neuss pendant presque un an. On observe un phénomène similaire en France, à la fin du règne de Charles V en 1370-1372, lorsqu'environ 15\% des lettres de rémission étant alors destinées à des gens d'armes. Sous Charles VI (1380-1422), ce taux diminue drastiquement à $2,4 \%$, sans doute du fait de la longue trêve francoanglaise de $1380-1415^{114}$. Charles VII (1422-1461) va quant à lui faire un usage intensif des lettres d'abolition afin de gracier les crimes commis par les routiers dans les dernières années de la guerre de Cent Ans ${ }^{115}$. C'est ici une différence importante de la politique miséricordieuse du roi de France, car dans les principautés bourguignonnes, l'abolition est un instrument essentiellement réservé aux villes rebelles à l'autorité du duc, une seule lettre de ce type destinée à des combattants ayant été trouvée dans les registres de l'audience ${ }^{116}$.

Afin de modifier la conduite des gens de guerre et promouvoir l'idéal d'un combattant professionnel et loyal, la législation militaire des ducs de Bourgogne désigne et réprime les crimes qui relèvent des excès de guerre, indignes des bons soldats, en les rendant même irrémissibles. À l'inverse, les lettres de rémission pardonnent majoritairement les actes qui relèvent des formes de violence socialement acceptée, tel que l'homicide non prémédité. Les gens de guerre, eux-mêmes, informés des attentes du pouvoir, font de leur demande de grâce la «chambre d'écho» de la propagande princière ${ }^{117}$. Ainsi, $85 \%$ des lettres du corpus bourguignon évoquent le service de guerre pour motiver la demande de grâce. Mais, au $X V^{e}$ siècle, le passé militaire ne suffit pas à établir l'image du combattant idéal, encore faut-il que celuici ait «bien et loyamment servy [...] sans oncquesmais avoir commis chose digne de reprehencion ${ }^{118} \gg$. De fait, dans environ 54\% des lettres, les gens de guerre appuient leur supplique en affirmant qu'ils possèdent une bonne réputation, ou «bonne fame et renommée», ce qui se traduit par l'absence de passé judiciaire («sans oncquesmais avoir esté actains ou convaincuz d'aucun autre vilain cas, blasme ou reprouche ${ }^{119} »$ ).

Au-delà de sa dimension purement disciplinaire visant à dicter les comportements individuels, le gouvernement des gens de guerre par la grâce peut également présenter un certain intérêt en termes de direction des armées. En accordant leur rémission au nom du service de guerre qu'ont presté les suppliants, les ducs de Bourgogne attendent également de ces derniers qu'ils continuent à les servir, comme l'indiquent régulièrement les formules de pardon: "pour consideration des bons services que icellui suppliant nous a faiz et esperons que encores faire doye, audit suppliant

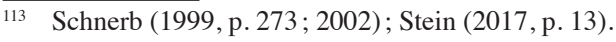

114 Gauvard (1991, p. 533).

115 Gauvard (2008).

116 ADN, B 1693 fol. 89 v.-99 r. (juillet 1469). Sur les abolitions accordées aux villes : Soen (2012).

117 Guyotjeannin (2010).

118 ADN, B 1698 fol. 15 r.-16 v. (août 1475).

119 ADN, B 1691 fol. 99 r.-101 r. (octobre 1466). Voir également Gauvard (2000, p. 290).
} 
inclinans a saditte supplicacion avons oudit cas quittié, remis et pardonné ${ }^{120} . »$ Par ailleurs, trois lettres de rémission signalent que, à la suite de leur crime, les suppliants ont déserté afin d'échapper à la justice ${ }^{121}$. La grâce mettant fin aux poursuites, les ducs de Bourgogne offrent la possibilité aux déserteurs d'un retour à l'armée. De même, les gens de guerre qui ont choisi de demeurer parmi les troupes se trouvent récompensés pour leur loyauté, à l'instar de Loysset Espessart, dont le capitaine «fut content que ledit suppliant demourast en notredit service durant ladicte armee» après qu'il ait commis son crime ${ }^{122}$. Il semble par ailleurs que les gens de guerre bénéficient d'une procédure de grâce accélérée: alors que, dans l'État bourguignon, le délai d'octroi d'une lettre de rémission est généralement supérieur à un an, plus de la moitié des grâces destinées à des combattants en service au moment de commettre leur crime sont délivrées entre un et six mois après les faits.

Enfin, la promesse d'une grâce peut être un moyen rapide de recruter des combattants. Ce phénomène est relativement bien connu dans l'Angleterre du $\mathrm{XIV}^{\mathrm{e}}$ siècle, où la Couronne publie régulièrement des proclamations royales qui promettent de gracier tous les fugitifs acceptant de rejoindre les armées. Certaines années, jusqu'à plusieurs centaines de soldats sont ainsi recrutés en échange d'une lettre de pardon ${ }^{123}$. S'il est vrai que l'on n'atteint pas des chiffres aussi élevés dans l'État bourguignon au XV siècle, le fait qu'une part importante des lettres de notre corpus ait été accordée à des fugitifs ayant rejoint les armées suggère tout de même que la promesse d'une grâce peut être un moyen rapide pour les ducs de Bourgogne de recruter des combattants. De plus, deux lettres de rémission, délivrées en 1474 et 1478 , signalent la promulgation de lettres patentes offrant la grâce aux bannis et homicides qui acceptent de servir en arme: «feismes publier en notre ville de Valencienne que tous ceulx qui pour mort d'hommes et autres sembables cas non enormes mais remissibles s'estoient absentez de nosdits pays et seignouries et avoient volenté de nous servir en armes es guerres lors et presentement regnans retournassent franchement et sans aucun dangier en nosdits pays ${ }^{124}$.» Or, nous avons vu précédemment que trois lettres imposent explicitement comme condition que le suppliant sera tenu «de nous servir en armes durant cesdites guerres et divisions ${ }^{125}{ }^{\text {» }}$. Cette exigence du service armé rejoint finalement la question de la sujétion de l'homme de guerre à l'autorité ducale: la lettre de rémission scelle un contrat implicite entre le duc de Bourgogne et ses combattants, le premier octroyant son pardon aux seconds en échange de leur obéissance.

Dans un article fondateur sur l'exercice de la justice dans les armées à la fin du Moyen Âge, Bertrand Schnerb affirme que «le problème de la justice appliquée aux gens de guerre à la fin du moyen âge montre un pouvoir princier oscillant entre deux

$\overline{120}$ ADN, B 1690 fol. 7 r.-v. (avril 1460).

121 ADN, B 1693 fol. 51 r.-52 v. (décembre 1468); B 1695 fol. 2 v.-3 r. (juin 1473); B 1695 fol. 75 r. (janvier 1475).

122 ADN, B 1694 fol. 76 v.-77 r. (décembre 1470).

123 Lacey (2009, p. 100-106); Villalon (2013).

124 ADN, B 1699 fol. 43 v.-44 r. (mars 1478). De même, la lettre B 1695 fol. 46 r.-v. (août 1474) expose que les suppliants «sont liberalement venus en notre service» à la suite de «lecres patentes qui ont esté publiees en noz villes et pays de par de ça ayans rappellez tous banniz et homicides qui nous vouldroient venir servir en armes et leurs promis baillier remission de leurs cas ».

125 ADN, B 1699 fol. 43 v.-44 r. (mars 1478). 
attitudes extrêmes : le laxisme et la rigueur ${ }^{126} \gg$. La volonté des ducs de Bourgogne de renforcer la discipline des soldats s'exprime d'abord par le développement d'une véritable législation militaire. Dans le prolongement des règlements de Jean sans Peur et de Philippe le Bon, les ordonnances de Charles le Hardi ciblent les infractions qui nuisent à la cohésion des troupes ou relèvent d'un usage illégitime de la force et de l'autorité par les mauvais éléments de l'armée. Toutefois, l'incrimination répétée des excès des gens de guerre contraste avec la difficulté réelle des pouvoirs judiciaires à réprimer efficacement la violence des soldats, parfois avec la complaisance des autorités militaires. Dans cette perspective, il paraîtrait a priori logique de conclure que l'octroi répété de lettres de rémission aux gens de guerre contribue à maintenir l'impunité des combattants, libres de se livrer à toutes sortes d'excès. Pourtant, l'examen des documents conservés dans les registres de l'audience suggère au contraire que l'usage du droit de grâce vis-à-vis des soldats se situe du côté de la rigueur plutôt que du laxisme: en pardonnant en priorité les gens de guerre qui font usage d'une forme licite et modérée de violence, tout en remettant les individus qui ont combattus les mauvais éléments de l'armée, les ducs agissent en cohérence avec le projet disciplinaire porté par leur réglementation.

Comme l'écrit Claude Gauvard, l'activité législative «consiste à ordonner et à punir, du moins en théorie, ceux qui enfreignent les ordonnances. Le pouvoir peut ainsi distinguer entre les bons et les mauvais hommes d'armes ${ }^{127} \gg$.

Si la législation militaire condamne les soldats indisciplinés, les lettres de rémission permettent au contraire de récompenser ceux qui font preuve d'obéissance et de loyauté envers le prince et qui commettent des crimes qui ne dépassent pas les limites d'une violence acceptable. La valorisation du service de guerre participe également de la reconnaissance du statut du combattant, puisque ce motif de grâce devient une sorte de dignité attachée à l'office des armes ${ }^{128}$. Enfin, la fonction pacificatrice des lettres de rémission apaise les conflits qui traversent les armées et permet de préserver les combattants en exercice, de rappeler ceux qui ont fui pour échapper à la justice, voire d'attirer de nouvelle recrues qui espèrent obtenir le pardon en se rapprochant du prince. Le pardon fonde alors la sujétion de l'homme de guerre à l'autorité ducale.

Quentin Verreycken

Centre d'histoire du droit et de la justice Rue du Poirier, 10, bte L4.06.01 B-1348 Louvain-la-Neuve quentin.verreycken@uclouvain.be

\footnotetext{
126 Schnerb (1990, p. 112).

127 Gauvard (2000, p. 289).

128 Deruelle (2009).
} 


\section{BIBLIOGRAPHIE}

\section{Sources inédites}

Archives départementales du Nord. Chambre des comptes (= ADN, B), n 1681-1703.

\section{Sources imprimées}

Champeaux, E., Ordonnances franc-comtoises sur l'administration de la justice (1343-1477), Dijon et Paris, Université de Dijon, 1912 (Revue bourguignonne, 22).

Crépin, A. (Ed.), Construire l'armée française. Textes fondateurs des institutions militaires, Turnhout, Brepols, 2006.

Eustache Descamps, Euvres complètes, Ed. Queux de Saint-Hilaire, A.-H.-E. de, Raynaud, G., 11 vol., Paris, Didot, 1878-1903.

Gollut, L., Les mémoires historiques de la république séquanoise et des princes de la FrancheComté de Bourgogne, Arbois, Auguste Javel, 1846.

Le Jouvencel par Jean de Beuil, Ed. Lecestre, L., 2 vol., Paris, Renouard, 1887-1889.

Mémoires pour servir à l'histoire de France et de Bourgogne, contenant un journal de Paris sous les regnes de Charles VI et de Charles VII, 2 vol., Paris, Chez Julien-Michel Gandouin et Pierre-François Giffart, 1729.

Ordonnances des roys de France de la troisième race, 22 vol., Paris, Imprimerie royale, 17231849.

Pégeot, P., Derniame, O., Hénin, M. (Ed.), Les lettres de rémission du duc de Lorraine René II (1473-1508), Turnhout, Brepols, 2013.

\section{Travaux}

Allmand, C., The war and the non-combatant, in Fowler, K. (Ed.), The Hundred Years War, Londres, St Martin's Press, 1971, p. 163-83.

Allmand, C., Changing views of the soldier in late medieval France, in Contamine, P., GiryDeloison, C. (Ed.), Guerre et société en France, en Angleterre et en Bourgogne, $X I V^{k}-X V^{E}$ siècle, Lille, Université Charles de Gaulle. Centre d'histoire de la région du Nord et de l'Europe du Nord-Ouest, 1991, p. 171-188.

Arnade, P., Prevenier, W., Honor, vengeance, and social trouble. Pardon letters in the Burgundian Low Countries, Ithaca, Cornell University Press, 2015.

Beaulant, R., Fonction et usage de la lettre de rémission chez les ducs de Bourgogne à la fin du Moyen Âge, Annales de Janua [En ligne], 3, 2015, URL: <http://annalesdejanua.edel. univ-poitiers.fr/index.php?id=922>.

Beaulant, R., Du gouvernement de l'individu au gouvernement des hommes. Les normes politiques dans les lettres de rémission des ducs de Bourgogne, in Gouverner les hommes, gouverner les âmes. Actes du XLVI Congrès de la SHMESP (Montpellier, 28-31 mai 2015), Paris, Publications de la Sorbonne, 2016, p. 297-307.

Bercé, Y.-M., La dernière chance. Histoire des suppliques du Moyen Âge à nos jours, Paris, Perrin, 2014.

Boltanski, A., Forger le «soldat chrétien». L'encadrement catholique des troupes pontificales et royales en France en 1568-1569, Revue historique, 669, 2014, p. 51-85.

Boone, M., Want remitteren is princelijck. Vorstelijk genaderecht en sociale realiteiten in de Bourgondische periode, in Liber amicorum Achiel de Vos, Evergem, Gemeentebestuur van Evergem, 1989, p. 53-59.

Bourin, M., Chevalier, B., Le comportement criminel dans les pays de la Loire moyenne, d'après les LETTRES de rémission (vers 1380 - vers 1450), Annales de Bretagne et des pays de l'Ouest, 88, 1981, p. 245-263. 
Bove, B., Violence extrême, rumeur et crise de l'ordre public : la tyrannie du bâtard de Vaurus, in Foronda, F., Barralis, C., Sère, B. (Ed.), Violences souveraines au Moyen Âge : travaux d'une école historique, Paris, PUF, 2010, p. 123-132.

Brusten, C., Les compagnies d'ordonnance dans l'armée bourguignonne, in Grandson 1476. Essai d'approche pluridisciplinaire d'une action militaire du $X V^{b}$ siècle, Lausanne, Centre d'histoire, 1976, p. 112-169.

Bubenicek, M., Entre rébellion et obéissance. L'espace politique comtois face au duc Philippe le Hardi (1384-1404), Genève, Droz, 2013.

Cauchies, J.-M., La désertion dans les armées bourguignonnes de 1465 à 1476, Revue belge d'histoire militaire, 22, 2, 1977, p. 132148.

Cauchies, J.-M., L'essor d'une législation générale pour les Pays-Bas bourguignons dans le dernier quart du XVe siècle: aperçu et suggestions, Publications du Centre européen d'études burgondo-médianes, 21, 1981, p. 59-70.

Cauchies, J.-M., État bourguignon ou états bourguignons ? De la singularité d'un pluriel, in Hoppenbrouwers, P. C. M., Janse, A., Stein, R. (Ed.), Power and Persuasion. Essays on the art of State building in honour of W. P. Blockmans, Turnhout, Brepols, 2010, p. 58-68.

Cazaux, L., Réglementation militaire royale et usage de la force dans le royaume de France (XIV $-\mathrm{XVI}^{\mathrm{e}}$ siècles), Inflexions. Civils et militaires : pouvoir dire, 13, 2010, p. 93-104.

Cazaux, L., La justice militaire et ses implications politiques sous Louis XI. Les papiers de Tristan L'Hermite, prévôt des maréchaux de France, au Trésor des chartes, in Destemberg, A., Potin, Y., Rosenblieh, E. (Ed.), Faire jeunesses, rendre justice. À Claude Gauvard, Paris, Publications de la Sorbonne, 2015, p. 133-147.

Challet, V., Tuchins et brigands des bois: communautés paysannes et mouvements d'autodéfense en Normandie pendant la guerre de Cent Ans, in Images de la contestation du pouvoir dans le monde normand ( $X^{e}-X V I I I^{e}$ siècle), Caen, Presses Universitaires de Caen, 2007, p. 135-146.

Chauvelays, J. de la, Mémoires sur la composition des armées de Charles le Téméraire dans les deux Bourgognes, Mémoires de l'Académie des sciences, arts et belles-lettres de Dijon, 5, 1878-1879, p. 138-369.

Chauvelays, J. de la, Les armées des trois premiers ducs de Bourgogne de la Maison de Valois, Mémoires de l'Académie des sciences, arts et belles-lettres de Dijon, 6, 1880, p. 18-335.

Contamine, P., Guerre, État et société à la fin du moyen âge. Etudes sur les armées des rois de France. 1337-1494, Paris-La Haye, Mouton, 1972.

Cornette, J., La révolution militaire et l'État moderne, Revue d'histoire moderne et contemporaine, 41, 4, 1994, p. 696-709.

Cruz Coelho, M. H. da, Les Cortes en temps de guerre - une médiation interactive entre le roi et les corps sociaux du royaume de Portugal aux $\mathrm{XIV}^{\mathrm{e}}$ et $\mathrm{XV}^{\mathrm{e}}$ siècles, Parliaments, Estates and Representation, vol. 21, $\mathrm{n}^{\circ} 1,2001, \mathrm{p} .37-56$.

Dauven, B., Rémission, pardon et abolition: typologie de la grâce princière en Brabant aux $\mathrm{XVI}^{\mathrm{e}}$ et XVII ${ }^{\mathrm{e}}$ siècles. Essai méthodologique, in Annaert, P. et Scholtes, T. (Ed.), Pardon, pénitence et réconciliation. Journées internationales d'histoire du droit et des institutions, Saint-Hubert, 14-15 mai 2010, Bruxelles, Archives générales du royaume, 2014, p. 73-86.

Davis, N. Z., Pour sauver sa vie. Les récits de pardon au XVI siècle, Paris, Seuil, 1988.

Deruelle, B., «Pour Dieu, le roi et l'honneur». Ethos chevaleresque, mérite et récompense au XVI ${ }^{\mathrm{e}}$ siècle, Hypothèses, vol. 12, 2009, p. 209-220.

De Schepper, H., Het gratierecht in het Bourgondisch-Habsburgse Nederland, 1384-1633, vorstelijk prerogatief en machtsmiddel, in Coppens, H., Van Honacker, K. (Ed.), Symposium over de centrale overheidsinstellingen van de Habsburgse Nederlanden. Tien bijdragen over de staat, de regering en de ambtenaren van de 16de tot de 18de eeuw, Bruxelles, 1995, p. 43-87. 
Dodd, G., Justice and Grace. Private petitioning and the English Parliament in the late Middle Ages, Oxford, Oxford University Press, 2007.

Dodd, G., Writing wrong: the drafting of supplications to the Crown in later fourteenthcentury England, Medium Aevum, 80, 2, 2011, p. 217-246.

Downing, B. M., The military revolution and political change: origins of democracy and autocracy in early modern Europe, Princeton, Princeton University Press, 1992.

Fargette, S., Rumeurs, propagande et opinion publique au temps de la guerre civile (14071420), Moyen Âge, 113, 2, 2007, p. 309-334.

Fassin, D., La supplique. Stratégies rhétoriques et constructions identitaires dans les demandes d'aide d'urgence, Annales. Histoire, Sciences Sociales, 55, 5, 2000, p. 955-981.

Foucault, M., Surveiller et punir. Naissance de la prison, Paris, Gallimard, 1975.

Fréminville, J. de, Les Écorcheurs en Bourgogne (1435-1445). Étude sur les compagnies franches au XV siècle, Dijon, Darantière, 1888.

Gauvard, C., «De grace especial». Crime, état et société en France à la fin du Moyen Âge, Paris, Publications de la Sorbonne, 1991.

Gauvard, C., Les clercs de la Chancellerie royale française et l'écriture des lettres de rémission aux XIV et $X^{\mathrm{e}}$ siècles, in Fianu, K. et Guth, D.J. (éds.), Écrit et pouvoir dans les chancelleries médiévales: espace français, espace anglais (Actes du colloque international de Montréal, 7-9 septembre 1995), Louvain-la-Neuve, FIDEM, 1997, p. 281-291.

Gauvard, C., Discipliner la violence dans le royaume de France aux XIVe et XVe siècles: une affaire d'État?, in Jaritz, G. (Ed.), Disziplinierung im Alltag des Mittelalters und der frühen Neuzeit (Krems, 1996), Vienne, Österreichische Akademie der Wissenschaften, 1999, p. 173-204.

Gauvard, C., Rumeur et gens de guerre dans le royaume de France au milieu du XV ${ }^{\mathrm{e}}$ siècle, Hypothèses, 1, 2000, p. 281-292.

Gauvard, C., Le roi de France et le gouvernement par la grâce à la fin du Moyen Âge: genèse et développement d'une politique judiciaire, in Millet, H. (Ed.), Suppliques et requêtes: le gouvernement par la grâce en Occident (XII ${ }^{e}-X V^{e}$ siècle), Rome, École française de Rome, 2003, p. 371-404.

Gauvard, C., Violence licite et violence illicite, in Violence et ordre public au Moyen Âge, Paris, Picard, 2005, p. 264-282.

Gauvard, C., De la requête à l'enquête: réponse théorique ou réalité politique, in Gauvard, C. (Ed.), L'enquête au Moyen Âge, Rome, École française de Rome, 2008, p. 429-458.

Gauvard, C., Pardonner et oublier après la Guerre de Cent Ans. Le rôle des lettres d'abolition de la chancellerie royale française, in Marcowitz, R., Paravicini, W. (Ed.), Pardonner et oublier? Les discours sur le passé après l'occupation, la guerre civile et la révolution, Munich, Verlag, 2009, p. 27-55.

Godding, P., La législation ducale en Brabant sous le règne de Philippe le Bon (1430-1467), Bruxelles, Académie royale de Belgique, 2006.

Goffman, E., Asiles, Paris, Éditions de Minuit, 1984.

Grummitt, D., Changing perceptions of the soldier in late medieval England, in Kleineke, H. (Ed.), The Fifteenth Century, vol. 10, Woodbridge, Boydell \& Brewer, 2011, p. 189-202.

Guillaume, H. L. G., Histoire de l'organisation militaire sous les ducs de Bourgogne, Bruxelles, Hayez, 1848.

Guignier, A., L'honneur du soldat. Éthique martiale et discipline guerrière dans la France des Lumières, Paris, Champ Vallon, 2014.

Gunn, S., Grummitt, D., Cools, H., War, State, and society in England and the Netherlands, 1477-1559, Oxford, Oxford University Press, 2007. 
Guyotjeannin, O., Entre persuasion et révélation: la rhétorique de la grâce à la chancellerie royale française (XIVe-XVe siècles), in Claustre, J., Mattéoni, O., Offenstadt, N. (Ed.), Un Moyen Âge pour aujourd'hui. Mélanges offert à Claude Gauvard, Paris, PUF, 2010, p. 88-96.

Hausmann, U. et Schreiber, T., Euer Kaiserlichen Majestät in untertänigster Demut zu Füßen. Das Kooperationsprojekt «Untertanensuppliken am Reichshofrat in der Regierungszeit Kaiser Rudolfs II. (1576-1612)», in Denzler, A., Franke, E. et Schneider, B. (Ed.), Prozessakten, Parteien, Partikularinteressen : Höchstgerichtsbarkeit in der Mitte Europas vom 15. bis 19. Jahrhundert, Berllin, De Gruyter, 2015, p. 71-96.

Heerma Van Voss, L. (Ed.), Petitions in Social History, Cambridge, Cambridge University Press, 2002.

Lachaud, F., L'Éthique du pouvoir au Moyen Âge. L'office dans la culture politique (Angleterre, vers 1150-vers 1330), Paris, Garnier Classiques, 2010.

Lacey, H., The royal pardon: access to mercy in fourteenth-century England, York, York Medieval Press, University of York, 2009.

Lawrence, D. R., The complete soldier. Military books and military culture in early Stuart England, 1603-1645, Leyde, Brill, 2009.

Lecuppre-Desjardin, É., Le Royaume inachevé des ducs de Bourgogne (XIVe-XVe siècles), Paris, Belin, 2016.

Lot, F., L'art militaire et les armées au Moyen Âge et dans le Proche Orient, 2 vol., Paris, Payot, 1946.

Maillard-Luypaert, M., Les suppliques de la Pénitencerie apostolique pour les diocèses de Cambrai, Liège, Thérouanne et Tournai (1410-1411), Bruxelles/Rome, Institut historique belge de Rome, 2003.

Millet, H. (Ed.), Suppliques et requêtes : le gouvernement par la grâce en Occident (XII ${ }^{e}-X V^{e}$ siècle), Rome, École française de Rome, 2003.

Muchembled, R., Les jeunes, les jeux et la violence en Artois au XVI ${ }^{\mathrm{e}}$ siècle, in Ariès, P. (Ed.), Les jeux à la Renaissance, Paris, Vrin, 1982, p. 563-578.

Muchembled, R., La violence au village. Sociabilité et comportements populaires en Artois du XV $V^{e}$ au XVII siècle, Turnhout, Brepols, 1989.

Nubola, C., Würgler, A. (Ed.), Suppliche e «gravamina» : politica, amministrazione, giustizia in Europe (secoli XIV-XVIII), Bologne, Il Mulino, 2002.

Nubola, C., Würgler, A. (Ed.), Praktiken des Widerstandes: Suppliken, Gravamina und Revolten in Europa (15.-19. Jahrhundert), Berlin, Duncker \& Humblot, 2006.

Oestreich, G., Neostoicism and the early modern State, Cambridge, Cambridge University Press, 1982.

Paresys, I., Aux marges du royaume. Violence, justice et société en Picardie sous François I ${ }^{\text {er }}$, Paris, Publications de la Sorbonne, 1998.

Parker, G., The «military revolution »-a myth?, in Rogers, C. J. (Ed.), The military revolution debate: readings on the military transformations of early modern Europe, Boulder, Westview, 1995 [1976], p. 37-54.

Parker, G., La révolution militaire. La guerre et l'essor de l'Occident, 1500-1800, Paris, Gallimard, 1993.

Petit-Dutaillis, C., Documents nouveaux sur les mours populaires et le droit de vengeance dans les Pays-Bas au XV siècle. Lettres de rémission de Philippe le Bon, Paris, Champion, 1908.

Prétou, P., Crime et justice en Gascogne à la fin du Moyen Âge, Rennes, Presses universitaires de Rennes, 2010.

Prétou, P., Les voisins contre la route: réactions et imprécations communautaires en Gascogne face aux bandes armées pendant la guerre de Cent ans, in Pépin, G., Lainé, F. 
et Boutoulle, F. (Ed.), Routiers et mercenaires pendant la guerre de Cent ans : hommage à Jonathan Sumption. Actes du colloque de Berbiguières (13-14 septembre 2013), Bordeaux, Ausonius Editions, 2016, p. 133-145.

Roberts, M., The military revolution, 1560-1660, in Rogers, C. J. (Ed.), The military revolution debate: readings on the military transformations of early modern Europe, Boulder, Westview, 1995 [1956], p. 13-35.

Sablon du Corail, A., L'État princier à l'épreuve. Financer et conduire la guerre pendant la crise de l'État bourguignon (1477-1493), Revue historique, 679, 3, 2016, p. 549-576.

Saul, N., A farewell to arms? Criticism of warfare in late fourteenth-century England, in Given-Wilson, C. (Ed.), Fourteenth Century England, vol. 2, Woodbridge, Boydell and Brewer, 2002, p. 131-146.

Santamaria, J.-B., Servir le prince et garder la loi de la ville. Les serments des officiers du duc de Bourgogne au sud des Pays-Bas (fin XIV milieu XV siècle), Histoire urbaine, 39, 1 , 2014, p. 85-103.

Schnerb, B., Un thème de recherche: l'exercice de la justice dans les armées des ducs de Bourgogne (fin XIVe-fin $\mathrm{XV}^{\mathrm{e}}$ s.), Publications du Centre européen d'études bourguignonnes, 30, 1990, p. 99-115.

Schnerb, B., Un monastère dans la guerre : l'abbaye du Mont-Saint-Éloi (fin XIVe-début XV ${ }^{\mathrm{e}}$ siècle), in Contamine, P. et Guyotjeannin, O. (Ed.), La guerre, la violence et les gens, vol. 2, Paris, Comité des travaux historiques et scientifiques, 1996, p. 101-117.

Schnerb, B., L'État bourguignon 1363-1477, Paris, Perrin, 1999.

Schnerb, B., "L'honneur de la maréchaussée». Maréchalat et maréchaux en Bourgogne des origines à la fin du XV siècle, Turnhout, Brepols, 2000.

Schnerb, B., Le recrutement social et géographique des armées des ducs de Bourgogne (13401477), Cahiers du CRHiDI, 18, 2002, p. 53-67.

Soen, V., La réitération de pardons collectifs à finalité politiques pendant la Révolte des PaysBas (1565-1598). Un cas d'espèce dans les rapports de force aux Temps Modernes ?, in Dauven, B. et Rousseaux, X. (Ed.), Préférant miséricorde à rigueur de justice. Pratiques de la grâce (XIII ${ }^{e}$-XVII ${ }^{e}$ siècles), Louvain-la-Neuve, Presses universitaires de Louvain, 2012, p. 97-122.

Spierenburg, P., Protestant attitudes to violence : the early Dutch Republic, Crime, History \& Societies, 10, 2, 2006, p. 5-31.

Stein, R., Magnanimous dukes and rising states: the unification of the Burgundian Netherlands, 1380-1480, Oxford, Oxford University Press, 2017.

Teboul, J., Combattre et parader. Des masculinités militaires plurielles, Terrains \& travaux, 27, 2, 2015, p. 99-115.

Texier, P., La rémission au XIV siècle: significations et fonctions, in La faute, la répression et le pardon. Actes du 107e congrès national des sociétés savantes, section de philologie et d'histoire jusqu'à 1610 (Brest, 1982), Paris, Comité des travaux historiques et scientifiques, 1984, p. 193-205.

Toureille, V., Vol et brigandage au Moyen Âge, Paris, PUF, 2006.

Troch, K., «Plein comme un pot !» Les cabarets et la boisson à Namur durant la première moitié du XVIII ${ }^{e}$ siècle, in Parmentier, I., Rousseaux, X. et Auspert, S. (Ed.), Buveurs, voleuses, insensés et prisonniers à Namur au 18 e siècle: déviance, justice et régulation sociale au temps des Lumières, Namur, Presses universitaires de Namur, 2012, p. 15-46.

Ulbricht, O., Supplikationen als Ego-Dokumente. Bittschriften von Leibeigenen aus der ersten Hälfte des 17. Jahrhunderts als Beispiel, in Schulze, W. (Ed.), Ego-Dokumente. Annäherung an den Menschen in der Geschichte, Berlin, Akademie Verlag, 1996, p. 149174. 
Vaultier, R., Le folklore pendant la guerre de Cent ans d'après les lettres de rémission du Trésor des chartes, Paris, Librairie Guénédaud, 1965.

Van Meerbeeck, L., Inventaire des archives des tribunaux militaires, Gembloux, Duculot, 1939.

Vaughan, R., Charles the Bold, the Last Valois Duke of Burgondy, Londres, Longman, 1973.

Verreycken, Q., «Pour nous servir en l'armée». Le gouvernement et le pardon des gens de guerre sous Charles le Téméraire, duc de Bourgogne (1467-1477), Louvain-la-Neuve, Presses universitaires de Louvain, 2014.

Verreycken, Q., Une oisiveté dangereuse. Vers une réglementation du «temps libre» dans les armées bourguignonnes sous Charles le Hardi (1471-1477), C@hiers du CRHIDI [En ligne], 38, 2016, URL: <http://popups.ulg.ac.be/1370-2262/index.php ?id=297>.

Verreycken, Q., Charles le Hardi a-t-il inventé la révolution militaire ? Les réformes des armées bourguignonnes de 1467 à 1477, in Delsalle, P., Docquier, G., Marchandisse, A., Schnerb, B. (Ed.), Pour la singuliere affection qu'avons a luy. Études bourguignonnes offertes à Jean-Marie Cauchies, Turnhout, Brepols, 2017a, p. 515-522.

Verreycken, Q., Le fait du prince ou de son administration? Les lettres de rémission des ducs de Bourgogne (1386-1482), Publications du Centre européen d'études bourguignonnes, 57, 2017b, p. 63-75.

Verreycken, Q., L'État de grâce. Guerre et usage du pardon en Angleterre, France et anciens Pays-Bas au XV siècle, thèse de doctorat en histoire, inédite, Université catholique de Louvain, Louvain-la-Neuve, 2018.

Villalon, L. J. A., «Taking the king's shilling» to avoid «the wages of $\sin$ » : royal pardons for military malefactors during the Hundred Years War, in Villalon, L. J. A. et Kagay, D. J. (Ed.), The Hundred Years War (part III). Further considerations, Leyde-Boston, Brill, 2013, p. 357-435.

Viltart, F., Exploitiez la guerre par tous les moyens! Pillages et violences dans les campagnes militaires de Charles le Téméraire (1466-1476), Revue du Nord, 380, 2, 2009, p. 473-490.

Vrolijk, M., Les avis au prince des juges locaux sur les lettres de rémission dans les provinces de Flandre, Hollande et Zélande (1531-1567), in Hoareau-Dodinau, J., Rousseaux, X. et Texier, P. (Ed.), Le pardon, Limoges, Presses universitaires de Limoges, 1999, p. 373-384 (Cahiers de l'Institut d'Anthropologie Juridique de Limoges 3).

Vrolijk, M., Recht door gratie. Gratie bij doodslagen en andere delicten in Vlaanderen, Holland en Zeeland (1531-1567), Hilversum, Verloren, 2004.

Wright, N.A., The Tree of Battles of Honoré Bouvet and the laws of war, in Allmand, C. (Ed.), War, literature, and politics in the late Middle Ages, Liverpool, Liverpool University Press, 1976, p. 12-31.

Würgler, A., Voices from among the «silent masses »: humble petitions and social conflicts in early modern central Europe, in Heerma Van Voss, L. (Ed.), Petitions in Social History, Cambridge, Cambridge University Press, 2002, p. 11-34. 